\author{
Métodos Sintéticos para a Preparação de Biarilas \\ Silva, T. B.; da Silva, F. C.; Ferreira, V. F.* \\ Rev. Virtual Quim., 2017, 9 (3), 1258-1284. Data de publicação na Web: 22 de maio de 2017 \\ http://rvq.sbq.org.br
}

\title{
Synthetics Methods for the Preparation of Biaryls
}

Abstract: The biaryl structural unit is often found in natural products, drugs and agrochemicals. Therefore, the formation of the carbon-carbon bond is of extreme importance in the synthetic planning of substances. Many methods of obtaining biaryls are described in the literature, however, nickel or palladium catalyzed cross-coupling methodologies, such as Kuma-Tamao-Corriu, Negishi, Migita-Kosug-Stille, SuzukiMiyaura and Hiyama are the most important and most used tools today. These reactions avoid the need for protection and deprotection of functional groups, as they are tolerant to most groups, such as: aldehydes, ketones, amino, nitriles, hydroxides, esters and nitro group. In this work, these cross-coupling methodologies and their main characteristics will be discuss.

Keywords: Cross coupling; transition metal; C-C coupling; nickel; palladium.

\section{Resumo}

A unidade estrutural biarila é frequentemente encontrada em produtos naturais, fármacos e agroquímicos. Portanto, a formação da ligação carbono-carbono é de extrema importância no planejamento sintético de substâncias. Muitos métodos de obtenção de biarilas são descritos na literatura, entretanto as metodologias de acoplamento cruzado catalisado por níquel ou paládio, como a de Kuma-TamaoCorriu, Negishi, Migita-Kosug-Stille, Suzuki-Miyaura e Hiyama, são as ferramentas mais importantes e as mais utilizadas atualmente. Essas reações evitam a necessidade de proteção e desproteção de grupos funcionais, pois são tolerantes a maioria dos grupos funcionais, tais como: aldeídos, cetonas, amino, nitrilas, hidróxidos, ésteres e grupo nitro. Neste trabalho, serão abordadas essas metodologias de acoplamento cruzado e suas características principais.

Palavras-chave: Acoplamento cruzado; metal de transição; acoplamento C-C; níquel; paládio.

* Universidade Federal Fluminense, Instituto de Química, Departamento de Química Orgânica, Campus do Valonguinho, CEP 24020-150, Niterói-RJ, Brasil.

M cegvito@vm.uff.br DOI: $\underline{10.21577 / 1984-6835.20170074}$ 


\section{Métodos Sintéticos para a Preparação de Biarilas \\ Thais de B. da Silva, Fernando de C. da Silva, Vitor F. Ferreira* \\ Universidade Federal Fluminense, Instituto de Química, Departamento de Química Orgânica, Campus do Valonguinho, CEP 24020-150, Niterói-RJ, Brasil. \\ * cegvito@vm.uff.br}

Recebido em 16 de fevereiro de 2017. Aceito para publicação em 11 de maio de 2017

\section{Introdução}

\section{Reação de Kumada-Tamao-Corriu}

3. Reação de Negishi

4. Reação de Migita-Kosug-Stille

5. Reação de Suzuki-Miayura

\section{Reação de Hiyama}

\section{Conclusão}

\section{Introdução}

Biarilas são substâncias contendo dois anéis arila ligados através de uma ligação simples e são fragmentos químicos frequentemente encontrados em produtos naturais, compostos biologicamente ativos e materiais orgânicos, sendo de grande importância na indústria química e farmacêutica. ${ }^{1-3}$ Muitas moléculas orgânicas com importantes propriedades biológicas ou físicas contém o fragmento biarila como parte de suas estruturas, entre estas pode-se citar o medicamento losartana ${ }^{4}(\mathbf{1})$, usado no tratamento da hipertensão arterial, o antiinflamatório felbinaco ${ }^{5}$ (2), o fármaco antitumoral imatinibe ${ }^{6}$ (3) e o produto natural vancomicina ${ }^{7}(4)$, um o antibiótico glicopeptídico (Figura 1).

A formação da ligação carbono-carbono é um tópico de extrema importância na síntese orgânica. ${ }^{8}$ Inúmeros métodos de síntese foram desenvolvidos para a preparação da classe de compostos biarilas e atualmente os métodos mais utilizados são reações de acoplamento cruzado (acoplamento C-M/C-X, $\mathrm{M}=$ metal, Esquema 1, rota $\mathrm{A}$ ) catalisada por metal de transição por exemplo, reações Kumada-Tamao-Corriu, ${ }^{9,10}$ Negishi, ${ }^{11}$ MigitaKosugi-Stille, ${ }^{12}$ Suzuki-Miyaura ${ }^{13}$ e Hiyama, ${ }^{14}$ e desde 1970 estas reações de acoplamento vem recebendo atenção considerável e são indispensáveis na química orgânica, na química medicinal, na indústria de eletrônicos e de materiais com propriedades especiais. ${ }^{15,16}$ É importante ressaltar que essa reação foi reconhecida pela Real Academia de Ciências da Suécia e conferiu o Prêmio Nobel aos pesquisadores Suzuki, Negishi e Heck em 2010. Outras estratégias de obtenção de biarilas que utilizam metal de transição $\left(\mathrm{M}_{\mathrm{T}}\right)$ como catalisadores são 
Silva, T. B. et al.

descritas na literatura, como: arilação oxidativa direta ou inserção em ligação $\mathrm{C}-\mathrm{H}$ (C-H/C-M, Esquema 1, rota B) e acoplamento cruzado desidrogenado ( $\mathrm{C}-\mathrm{H} / \mathrm{C}-\mathrm{H}$, Esquema 1, rota $\mathrm{C}){ }^{17}$<smiles>CCCCc1nc(Cl)c(CO)n1Cc1ccc(-c2ccccc2-c2nn[nH]n2)cc1</smiles><smiles>Cc1ccc(NC(=O)c2ccc(CN3CCN(C)CC3)cc2)cc1Nc1nccc(-c2cccnc2)n1</smiles>

3<smiles>O=C(O)Cc1ccc(-c2ccccc2)cc1</smiles>

2

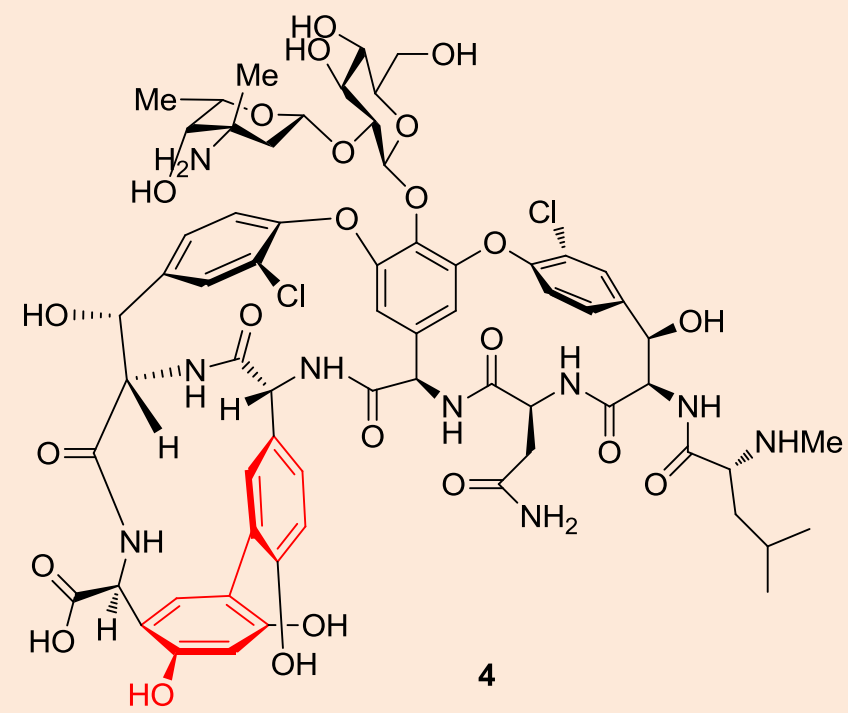

Figura 1. Fármacos que contém a porção biarila

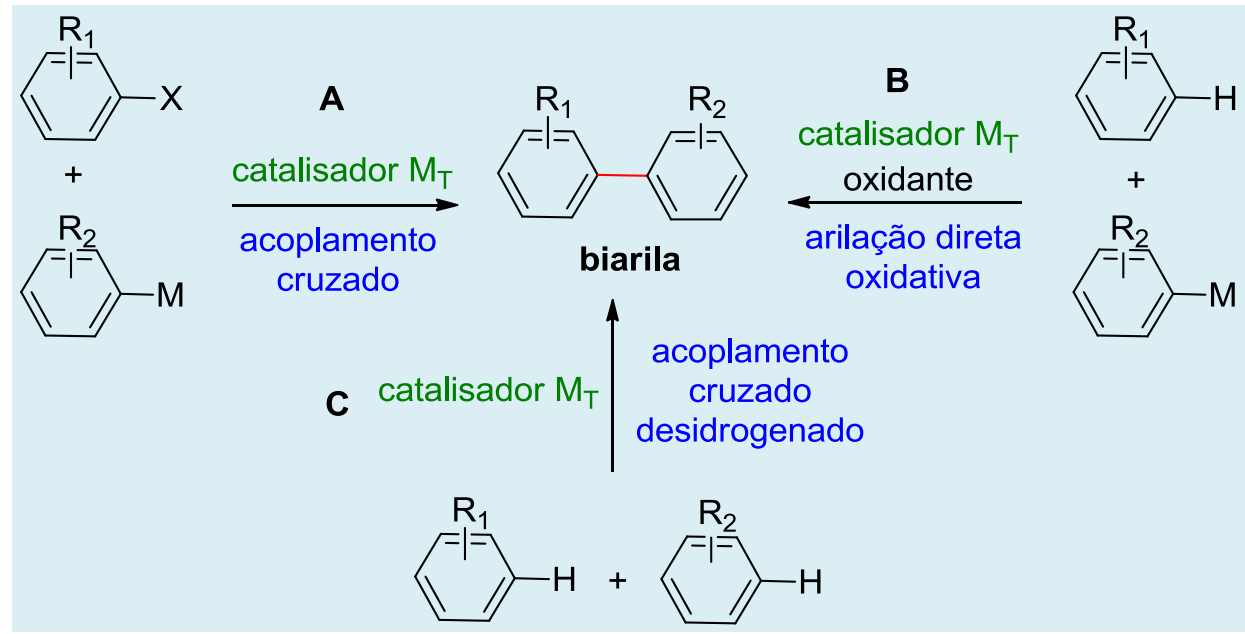

Esquema 1. Métodos de obtenção de biarilas por acoplamento ${ }^{18}$ 
As reações de acoplamento cruzado de halogenetos de arila utilizam reagentes de Grignard (acoplamento de Kumada) catalisada por níquel ou paládio, ${ }^{18}$ compostos de zinco (acoplamento de Negishi), ${ }^{11}$ compostos de organoestanhos (acoplamento de Stille), ${ }^{12}$ ácidos aril borônicos (acoplamento de Suzuki) ${ }^{13}$ e compostos de silício (acoplamento de Hiyama) (Esquema 2). ${ }^{14,19}$

Estas metodologias de acoplamento cruzado com metais de transição $\left(\mathrm{M}_{\mathrm{T}}\right)$ são classificadas com base no metal do grupo principal $\left(M_{P}\right)$, que é utilizado para transferir $\mathrm{R}^{\prime}$ na etapa de transmetalação. ${ }^{18} \mathrm{Em}$ geral, todas as reações de acoplamento cruzado têm mecanismo semelhante e a única diferença está na natureza do substrato. As etapas do mecanismo são adição oxidativa, transmetalação e eliminação redutiva (Esquema 3).

$$
\mathrm{R}-\mathrm{X}+\mathrm{R}^{\prime}-\mathrm{M}_{\mathrm{P}} \stackrel{\mathrm{M}_{\mathrm{Tcat}}}{\longrightarrow} \mathrm{R}-\mathrm{R}^{\prime}+\mathrm{M}_{\mathrm{P}-\mathrm{X}}
$$

$$
\begin{array}{ll}
X=\mathrm{I}, \mathrm{Br}, \mathrm{Cl}, \mathrm{OTs}, \mathrm{N}_{2} \mathrm{BF}_{4} \quad \begin{array}{l}
\mathrm{R}=\text { arila, alquila ou vinila } \\
\mathrm{R}
\end{array}=\text { alquila, arila ou vinila }
\end{array}
$$

\begin{tabular}{cccc}
\hline Método & Metal de transição $\left(\mathbf{M}_{\mathbf{T}}\right)$ & Metal do grupo principal $\left(\mathbf{M}_{\mathbf{P}}\right)$ & Referência \\
Kumada & $\mathrm{Pd} \mathrm{ou} \mathrm{Ni}$ & $\mathrm{MgX}, \mathrm{Li}$ & {$[9,10]$} \\
Negishi & $\mathrm{Pd} \mathrm{ou} \mathrm{Ni}$ & $\mathrm{Al}(i-\mathrm{Bu})_{2}, \mathrm{ZrClCp}_{2}, \mathrm{ZnX}$ & {$[11]$} \\
Stille & $\mathrm{Pd}$ & $\mathrm{SnR}_{3}$ & {$[12]$} \\
Suzuki & $\mathrm{Pd}$ & $\mathrm{BX}_{2}$ & {$[13]$} \\
Hiyama & $\mathrm{Pd}$ & $\mathrm{SiR}_{3}$ & {$[14]$} \\
\hline
\end{tabular}

Esquema 2. Esquema geral das reações de acoplamento cruzado catalisadas por metal de transição

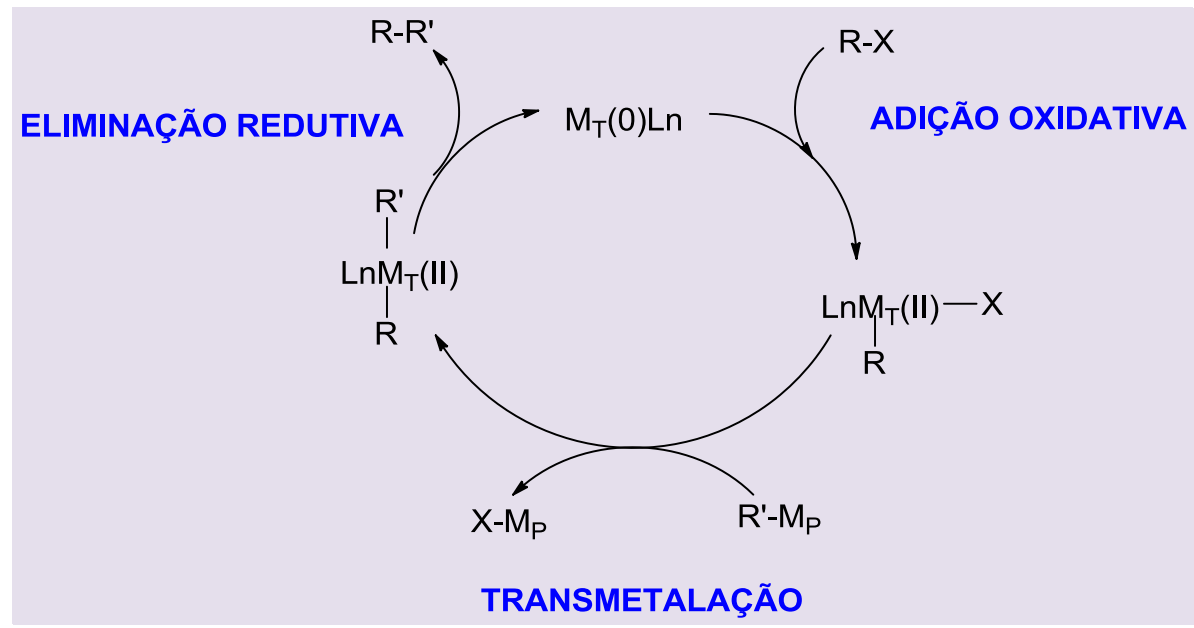

Esquema 3. Mecanismo geral para as reações de acoplamento cruzado

As reações de acoplamento carbonocarbono, com exceção do acoplamento de Kumada, funcionam com reagentes contendo grupos aldeídos, cetonas, amino, nitrilas, hidróxidos, ésteres nitro, e por isso, não há necessidade de proteção e desproteção de 
grupos funcionais durante estas reações. ${ }^{20}$

Neste trabalho serão abordadas as principais e mais utilizadas reações de acoplamento cruzado catalisadas por metal de transição para formação da ligação carbono-carbono e obtenção de biarilas.

\section{Reação de Kumada-Tamao-}

\section{Corriu}

Desde a década de 40, já se sabia que reagentes de Grignard acoplavam entre si por ação de halogenetos metálicos, através de uma reação de homoacoplamento do composto de Grignard, possibilitando obterse biarilas simétricas. ${ }^{18}$ Entretanto, apesar destas reações levarem a misturas de produtos de homoacoplamento, ainda assim, os reagentes de Grignard não poderiam ser descartados, já que são os mais disponíveis comercialmente. No entanto, isto impulsionou novas pesquisas a fim de melhorar estas metodologias.
Em 1971, Kochi e Tamura ${ }^{21}$ descreveram uma reação de acoplamento cruzado catalisada por metal de transição (Ag, Fe ou $\mathrm{Cu}$ ) envolvendo halogenetos orgânicos e reagente de Grignard, porém os problemas com a obtenção de subprodutos ainda não tinham sido solucionados, pois nesta reação eram obtidos produtos de homoacoplamento e acoplamento cruzado.

Paralelamente e de forma independente, em 1972 Kumada, Tamao $^{10}$ e Corriu $^{10}$ desenvolveram uma metodologia de acoplamento cruzado entre halogenetos de alquila, arila ou vinila e reagentes de Grignard catalisado por complexos de níquel. Desde então, catalisadores de níquel e paládio tornaram-se os mais utilizados e a reação de Kumada-Corriu-Tamao deu início a novas metodologias de formação da ligação C-C catalisadas por metais de transição. ${ }^{20}$

Em 1984, Kumada e Tamao descreveram a síntese do produto (7), usando 2,6cloropiridina (5) e dois reagentes de Grignard diferentes, catalisada de paládio (Esquema 4). ${ }^{22,23}$
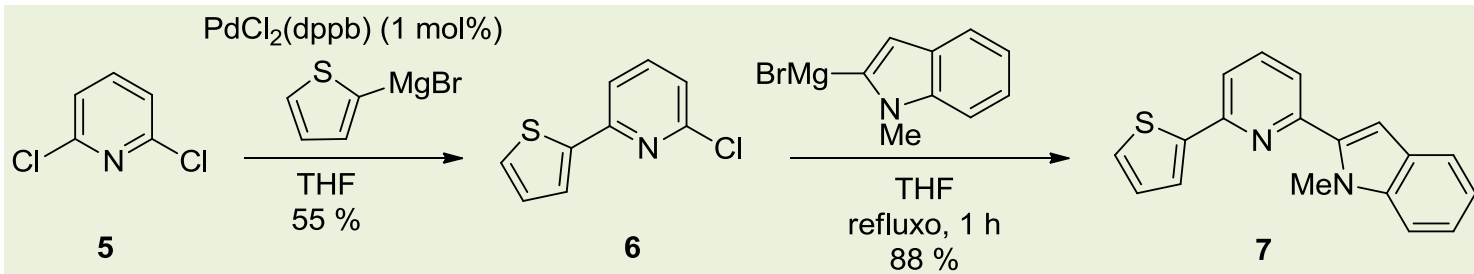

Esquema 4. Acoplamento de Kumada-Tamao-Corriu, usando 2,6-cloropiridina e dois reagentes de Grignard heteroaril diferentes

Recentemente, Zhang e colaboradores sintetizaram uma série de biarilas (10a-e) através do acoplamento de Kumada (Esquema 5) a partir de cloretos de arila e aril Grignard em THF como solvente e catalisada por níquel(II) contendo carbenos (NHCs), que se mostrou bastante eficiente, pois com apenas $0,5 \mathrm{~mol} \%$ do complexo, os produtos de acoplamento foram obtidos com excelentes rendimentos. Os substratos $p$ - diclorobenzeno e o-diclorobenzeno geraram apenas o produto monosubstituído (10c e 10d), já para o $m$-diclorobenzeno, foi observado uma mistura de produtos mono- e di-substituídos sob as condições iniciais. Com o aumento da quantidade de reagente de Grignard para 2,5 mmol e o catalisador para 1 mol\%, detectou-se o produto $m$-terfenil (10e) com $98 \%$ de rendimento. ${ }^{24}$ 


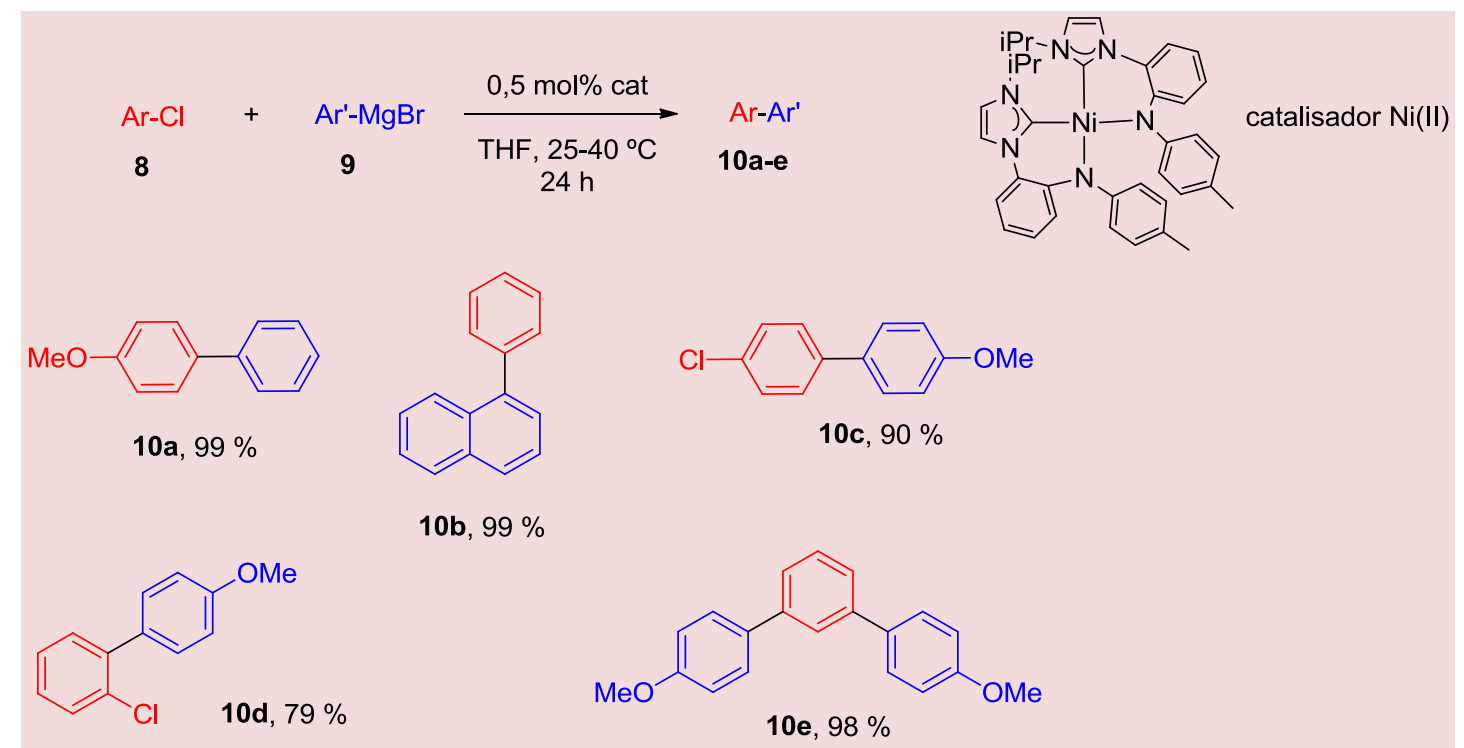

Esquema 5. Acoplamento de Kumada catalisado por complexos de níquel carbenos (NHCs)

Vários derivados biarilados (13a-c) foram sintetizados através do acoplamento de Kumada catalisado por um complexo base de Schiff de níquel(II). Kuchtanin e colaboradores demostraram a eficiência deste catalisador nesta reação, obtendo-se os produtos com bons rendimentos (Esquema 6). ${ }^{25}$

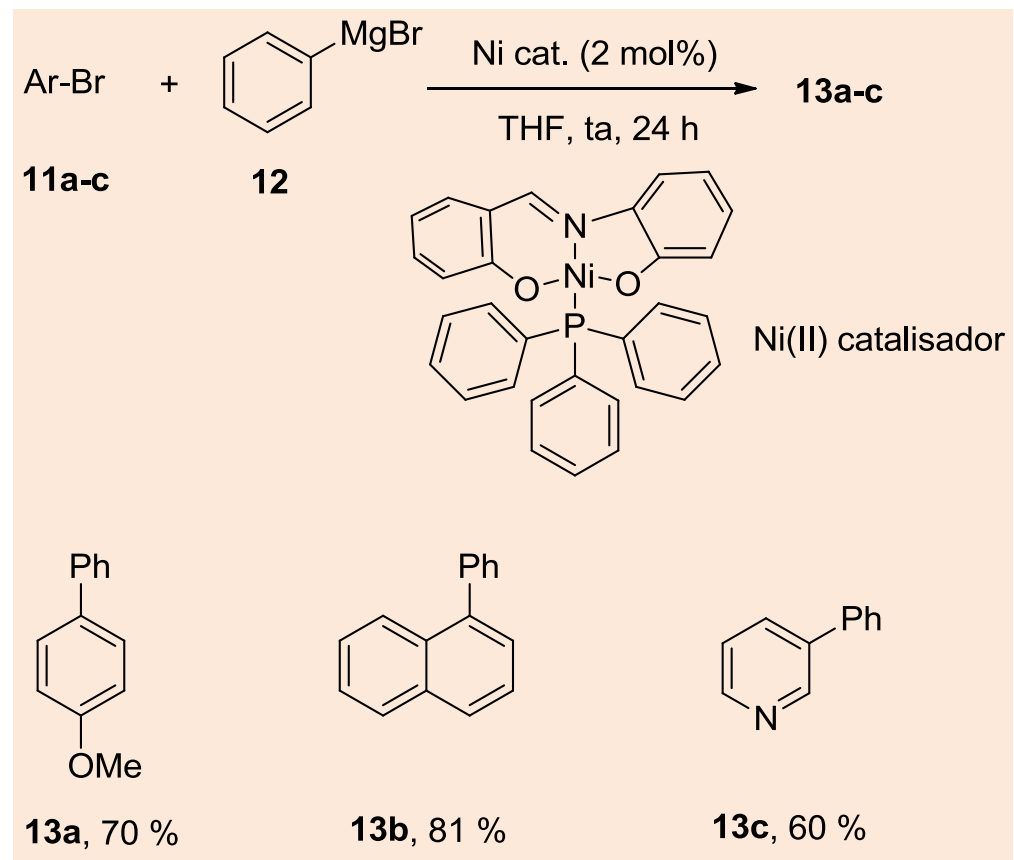

Esquema 6. Acoplamento de Kumada catalisado por um complexo base de Schiff de níquel(II)

O atazanavir (17) é um fármaco inibidor da protease do vírus HIV utilizado no tratamento da AIDS. A sua cadeia lateral contém o grupo 4-piridi-2-il-benzaldeído (16), que foi obtido através da reação de Kumada entre o reagente de Grignard (14) e a 2bromopiridina (15), catalisada por $\mathrm{Ni}(\mathrm{dppp}) \mathrm{Cl}_{2}$. O reagente DIBALH foi 
adicionado à reação com o objetivo de reduzir o $\mathrm{Ni}(\mathrm{II})$ a $\mathrm{Ni}(0)$ para dar início ao novo ciclo catalítico (Esquema 7). ${ }^{26}$

Stein e colaboradores descreveram a reação de acoplamento cruzado de organoselenetos (18) insaturados com reagente de Grignard, através da reação de Kumada catalisada por $\mathrm{Pd}\left(\mathrm{PPh}_{3}\right)_{4}$, obtendo-se os produtos 19a-b com bons rendimentos (Esquema 8). ${ }^{27}$<smiles>CC(O)c1ccc(N(Br)Br)cc1</smiles>

(14)<smiles>Brc1ccccn1</smiles>

(15)

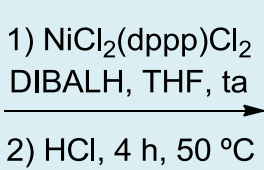

2) $\mathrm{HCl}, 4 \mathrm{~h}, 50^{\circ} \mathrm{C}$

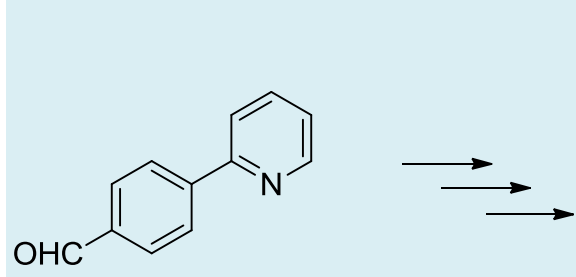

$16,90 \%$<smiles>COC(=O)N[C@H](C(=O)N[C@@H](Cc1ccccc1)[C@H](O)CN(Cc1ccc(-c2ccccn2)cc1)NC(=O)[C@H](NC(=O)OC)C(C)(C)C)C(C)(C)C</smiles>

Esquema 7. Uso da reação de Kumada na síntese do atazanavir (17)

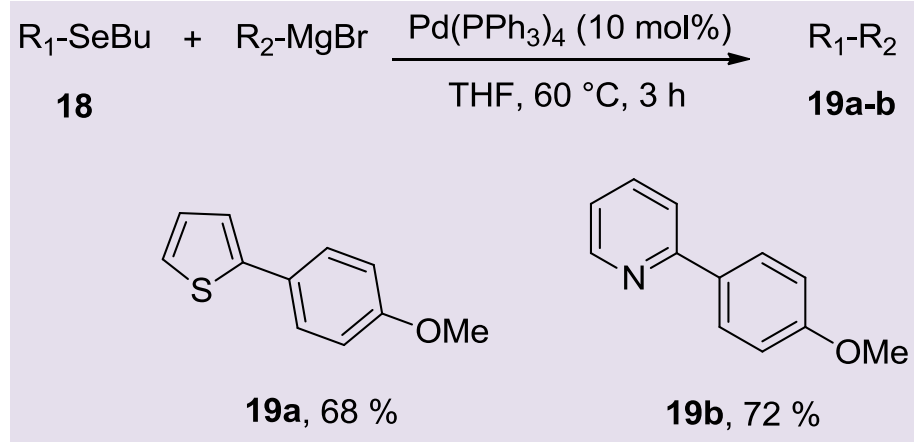

Esquema 8. Reação de Kumada usando organoselenetos

O honokiol (23) é um neolignano biarílico não simétrico, extraído das plantas medicinais Magnoliae officinalis e Magnoliae abovota, tem grande importância na medicina por apresentar atividades biológicas como antidepressivo, antibacteriano, antiviral, anticancerígeno, anti-inflamatório. A síntese total do honokiol (23), com rendimento global de $68 \%$, foi descrita por Srinivas e colaboradores e teve como etapa chave o acoplamento de Kumada do 2bromoanisol (20) com o 4-iodoanisol (21) catalisado por $\mathrm{Pd}\left(\mathrm{PPh}_{3}\right)_{4}$, formando $\mathrm{O}$ intermediário biarilado (22) com $85 \%$ de rendimento (Esquema 9). ${ }^{28}$

A reação de acoplamento cruzado de Kumada foi empregada por Ackermann e colaboradores na síntese de biarilas a partir de aril e heteroaril tosilatos. (Esquema 10). ${ }^{29}$ 


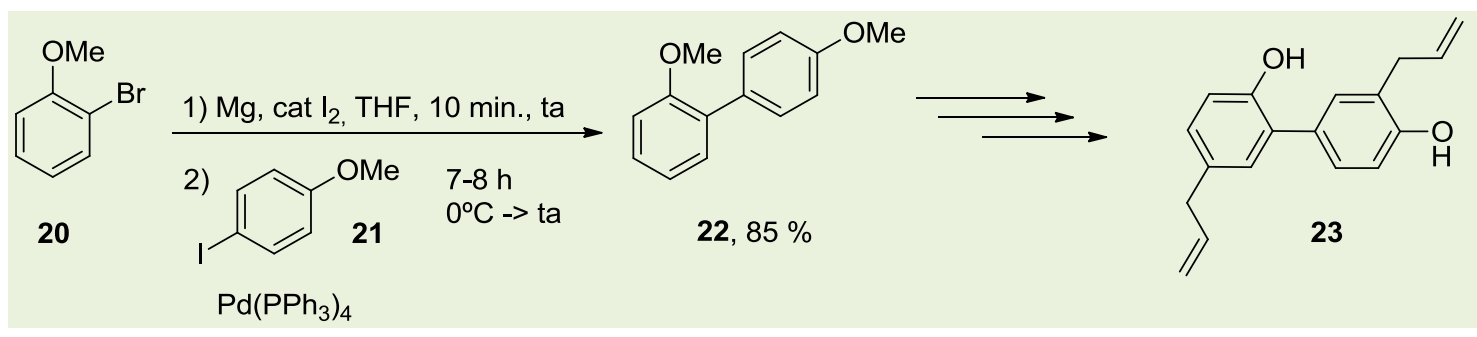

Esquema 9. Acoplamento de Kumada empregado na síntese do honokiol 23

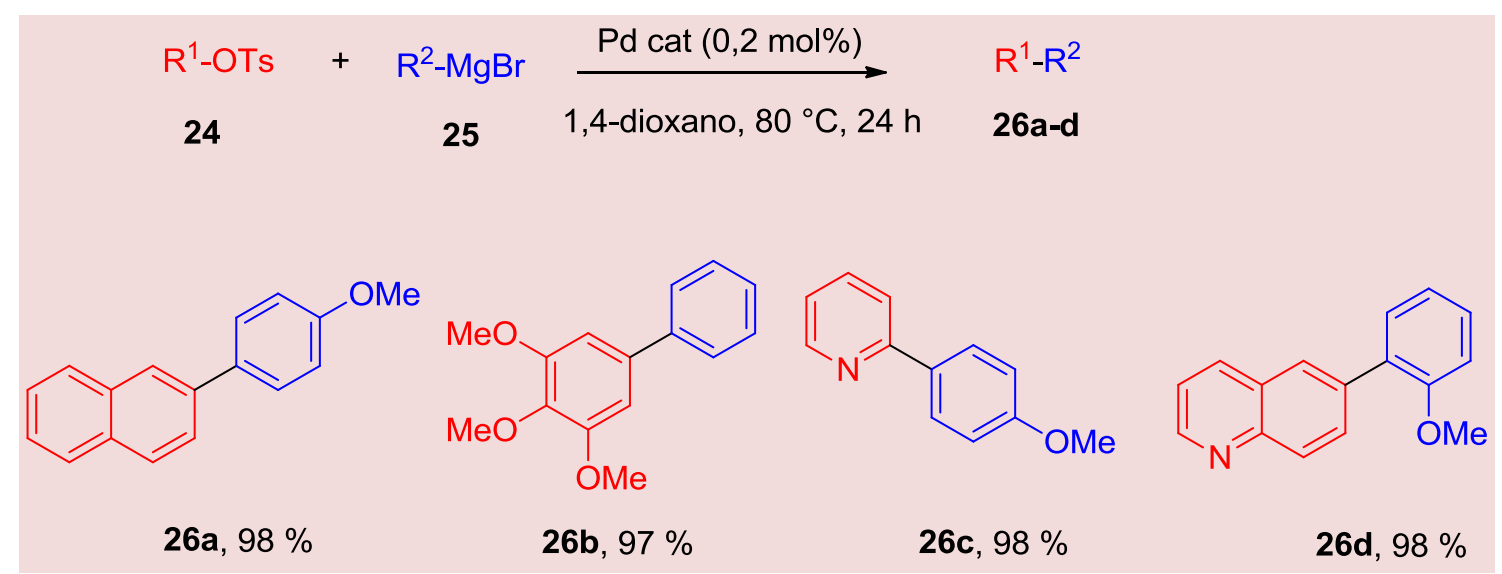

Esquema 10. Acoplamento de Kumada entre aril e heteroaril tosilatos e reagentes de Grignard

O acoplamento de Kumada é empregado na síntese de biarilas assimétricas e desde 1975 foram publicados vários estudos sobre a possibilidade do seu uso em acoplamento cruzado assimétrico catalisado por níquel ou paládio. Em seu primeiro estudo de reações assimétricas, Kumada e Tamao utilizaram um ligante fosfina quiral $(R, R)$-(-)-diop com níquel para acoplar reagentes de Grignard arilsubstituídos com halogenetos de arila, obtendo-se a binaftila 29 (Esquema 11). ${ }^{30}$

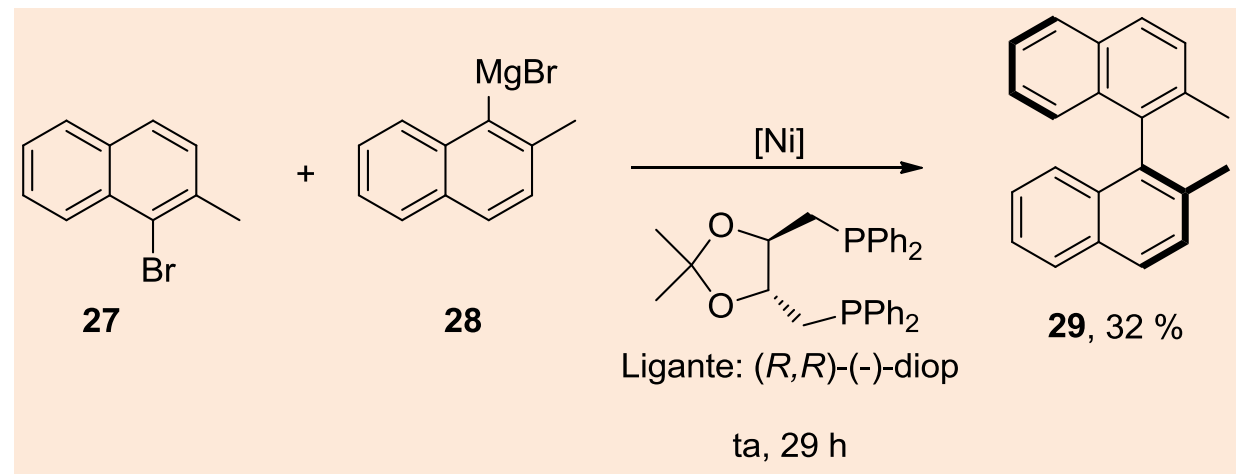

Esquema 11. Primeiro acoplamento assimétrico aril-aril através da reação de Kumada

Em 2013, Wu e colaboradores relataram a síntese de biarilas assimétricas utilizando um ligante quiral monodentado carbeno $\mathrm{N}$ heterocíclico para a reação de Kumada catalisada por paládio. Nesta reação, o brometo de 2-metoxinaftilmagnésio (30) gerado in situ é acoplado ao 1bromonaftaleno (31), conduzindo ao produto 
quiral binaftila (32) em $81 \%$ de rendimento e $48 \%$ de excesso enantiomérico (Esquema 12). O complexo de paládio (aS-aS) mostrou- se bastante eficiente e com moderada enantioseletividade. ${ }^{31}$

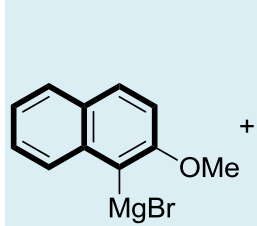

30<smiles>Brc1cccc2ccccc12</smiles>

31

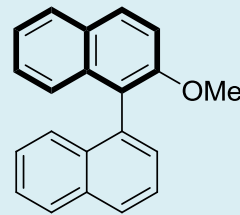

32

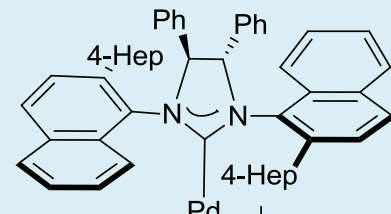

$\mathrm{Cl}^{\prime}$

$$
81 \%, 48 \% \text { ee (aS-aS) }
$$

Esquema 12. Acoplamento de Kumada com catalisador quiral, um complexo de paládio-NHC

\section{Reação de Negishi}

Em 1977, foi publicada a primeira reação do acoplamento cruzado de Negishi. ${ }^{12}$ Esta reação tem uma vantagem sintética que é a utilização de reagentes organozinco, os quais são tolerantes a uma grande variedade de grupos funcionais, além disso, estes reagentes podem ser preparados a partir da reação de halogenetos orgânicos com zinco metálico.
Uma variedade de biarilas funcionalizadas foram sintetizados por Wang e colaboradores, utilizando reagentes de organozinco, que foram preparados previamente pelo tratamento de halogenetos de arila com pó de zinco e $\mathrm{LiCl}$. A reação de acoplamento foi catalisada por PEPPSI (do inglês Pyridine-Enhanced Precatalyst Preparation Stabilisation and Initiation), um catalisador à base de paládio contendo o núcleo piridina. Deste estudo, destacam-se os produtos $35 a, 35 b, 35 c$, os quais foram obtidos em maiores rendimentos (Esquema 13). ${ }^{32}$

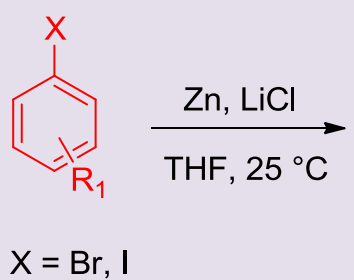

33

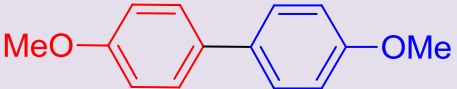

$35 a, 95 \%$<smiles>[R]c1cccc([R4])c1</smiles>

34
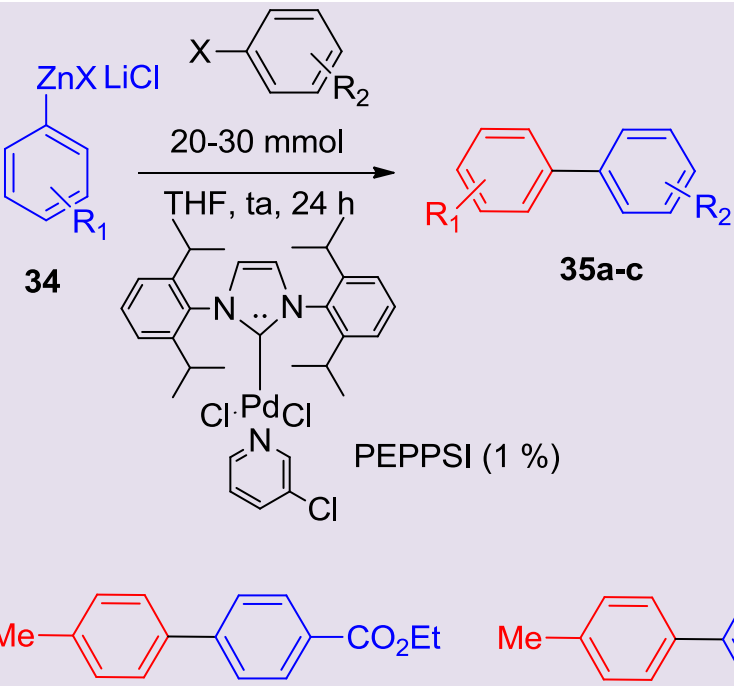

$35 b, 90 \%$

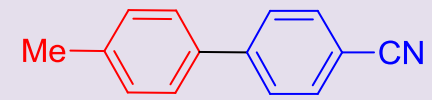

35c, $94 \%$

Esquema 13. Acoplamento de Negishi catalisado por PEPPSI 
Recentemente, o acoplamento de Negishi foi empregado por Roesner e colaboradores na síntese de biarilas, partindo de fluorobenzeno e brometos de (hetero)arila em uma reação regiosseletiva, onde as etapas de metalação e acoplamento cruzado de Negishi são realizadas em reator de fluxo contínuo (Esquema 14). ${ }^{33}$

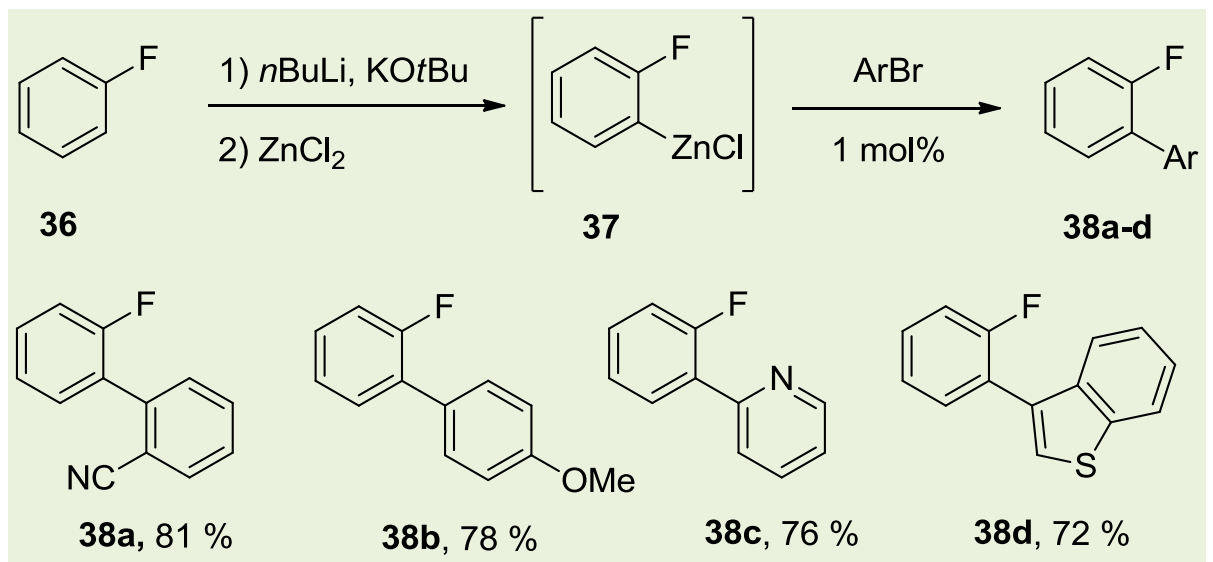

Esquema 14. Acoplamento de Negishi entre fluorobenzeno e brometos de (hetero)arila

A metodologia de acoplamento cruzado de Negishi foi usada para a produção do adapaleno (42), um retinoide sintético utilizado para o tratamento tópico da acne. Em sua síntese, o reagente de Grignard (39) reage com $\mathrm{ZnCl}_{2}$, em seguida acopla com o 6bromo-2-naftoato de metila (40) na presença do catalisador $\mathrm{NiCl}_{2}$ (dppe), formando $\mathrm{o}$ intermediário biarila (41) (Esquema 15). ${ }^{34}$

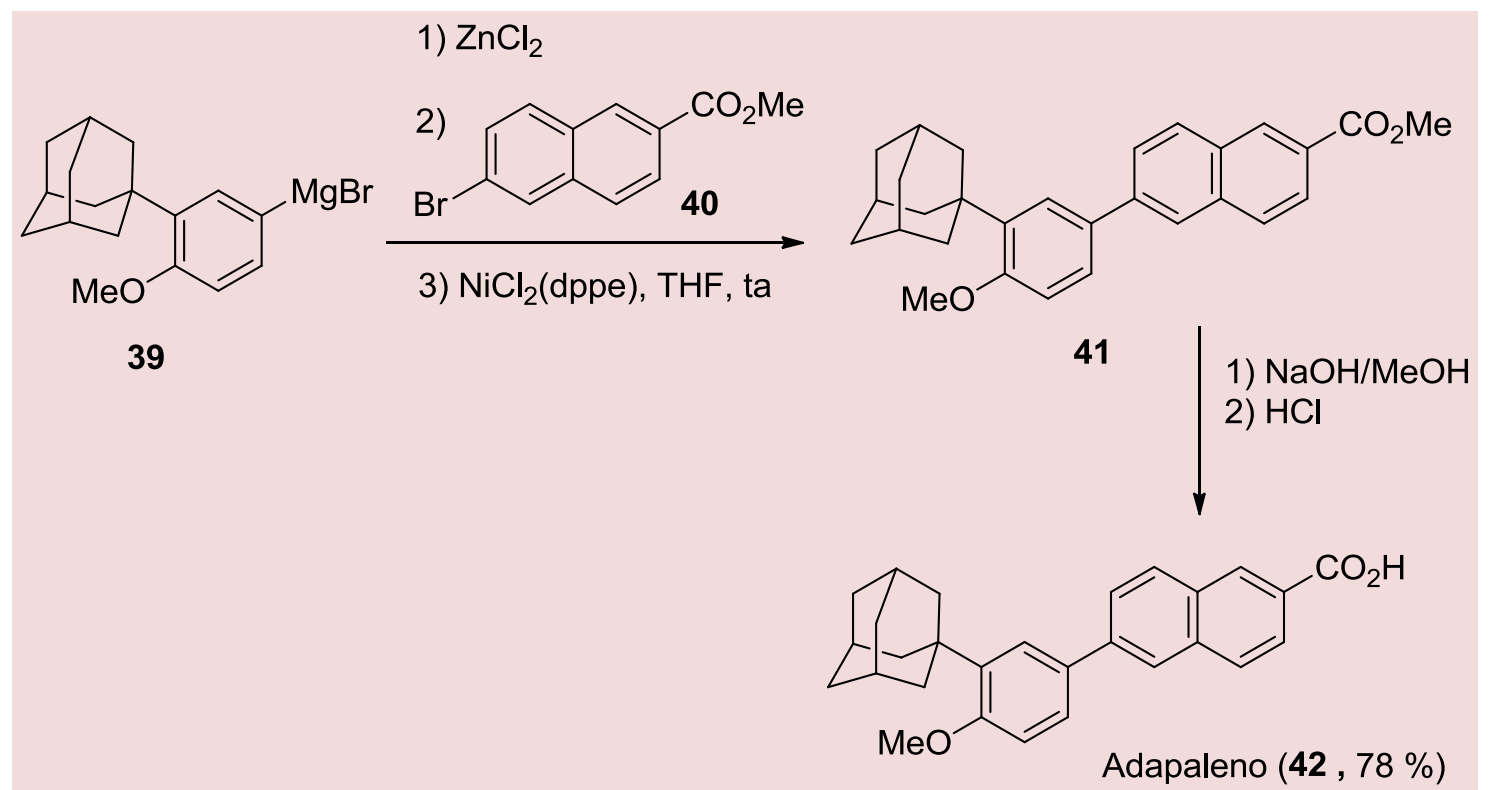

Esquema 15. Acoplamento de Negishi empregado na síntese do adapaleno

Wu e colaboradores descreveram o acoplamento cruzado de Negishi entre brometos de arila e cloretos de organozinco catalisado por um complexo de paládiobipiridila. As reações prosseguiram em condições brandas e foram bastante 
eficientes, gerando os produtos desejados em excelentes rendimentos (Esquema 16). ${ }^{35}$

Recentemente, Thapa e colaboradores descreveram um método de síntese de biarilas, utilizando o acoplamento de Negishi catalisado por iodeto de cobre (I) entre os reagentes arilzinco e iodetos de arila. Esta reação se processa sob condições livres de ligante e baixa carga de catalisador ( $5 \mathrm{~mol} \%$ ), e é tolerante para uma variedade de grupos funcionais (Esquema 17). ${ }^{36}$

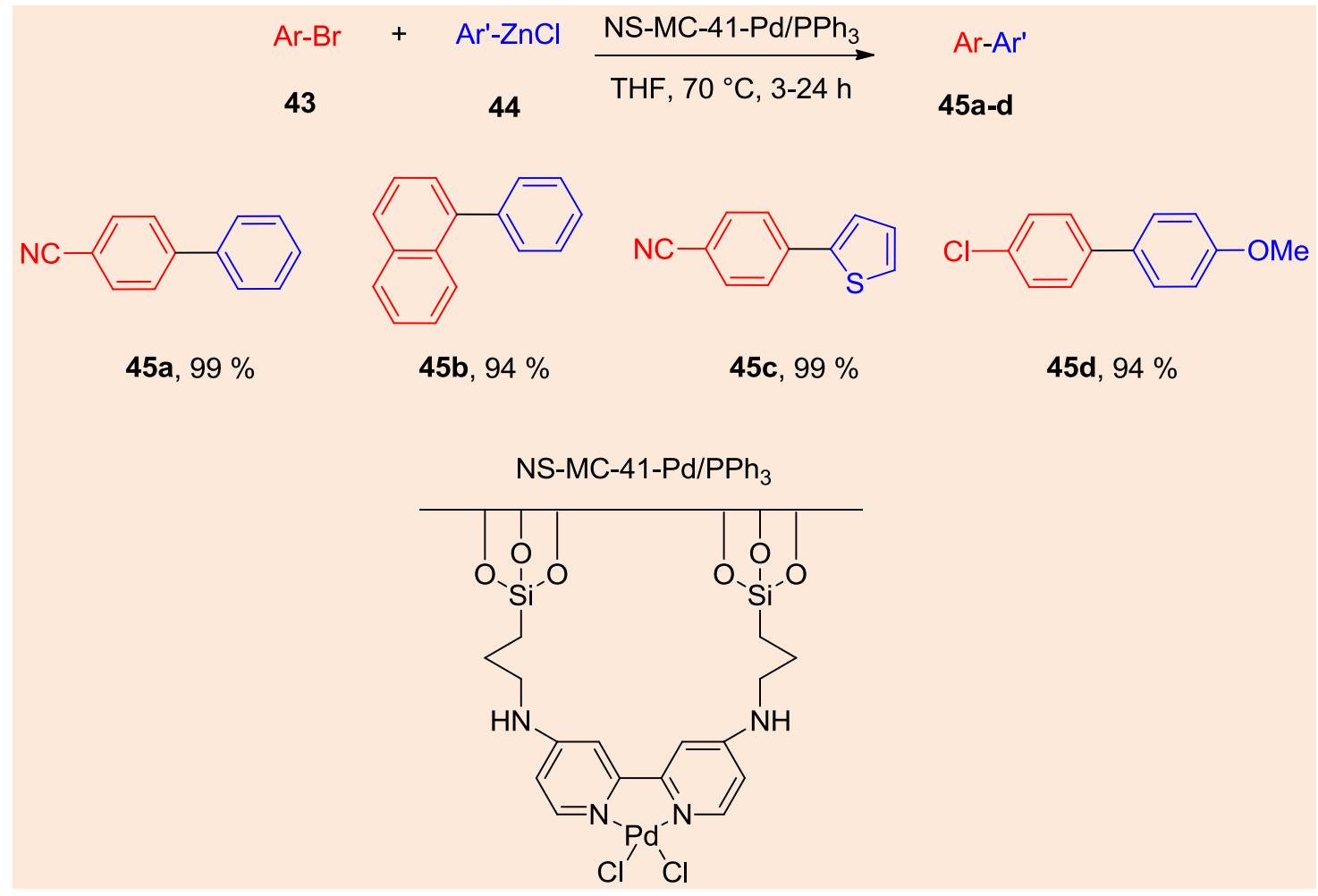

Esquema 16. Acoplamento de Negishi entre brometos de arila e cloretos de organozinco

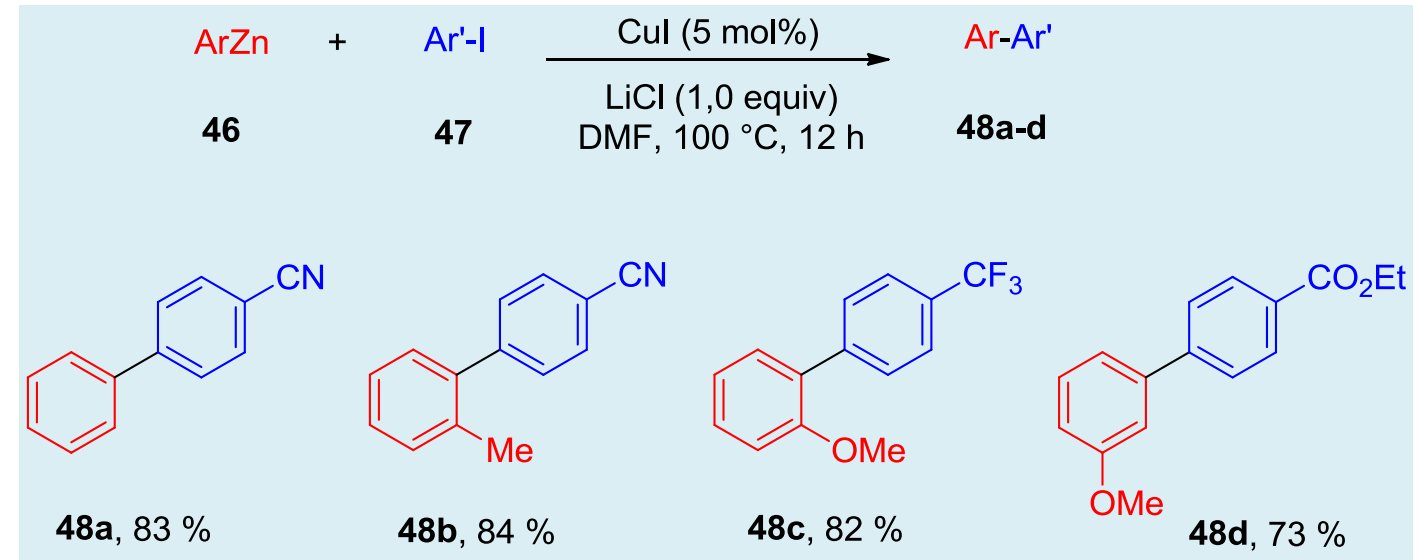

Esquema 17. Acoplamento de Negishi catalisado por iodeto de cobre (I)

Sieber e colaboradores desenvolveram uma síntese eficiente de biarilas, empregando o acoplamento cruzado de
Negishi catalisado por de $\mathrm{Pd}_{2}(\mathrm{dba})_{3} / \mathrm{BI}$-DIME na presença de ligantes di-hidrobenzooxafosfóis. As biarilas foram obtidas em 
ótimos rendimentos, conforme apresentado no Esquema $18 .^{37}$

Em 2011, o acoplamento de Negishi foi utilizado na preparação de biarilas a partir de diaril-teluretos em reação catalisada por paládio e cobre (Esquema 19). A vantagem desse protocolo está na transferência de ambos os anéis aromáticos ligados ao átomo de telúrio. Essa também é uma metodologia eficaz para a obtenção de compostos assimétricos. ${ }^{38}$

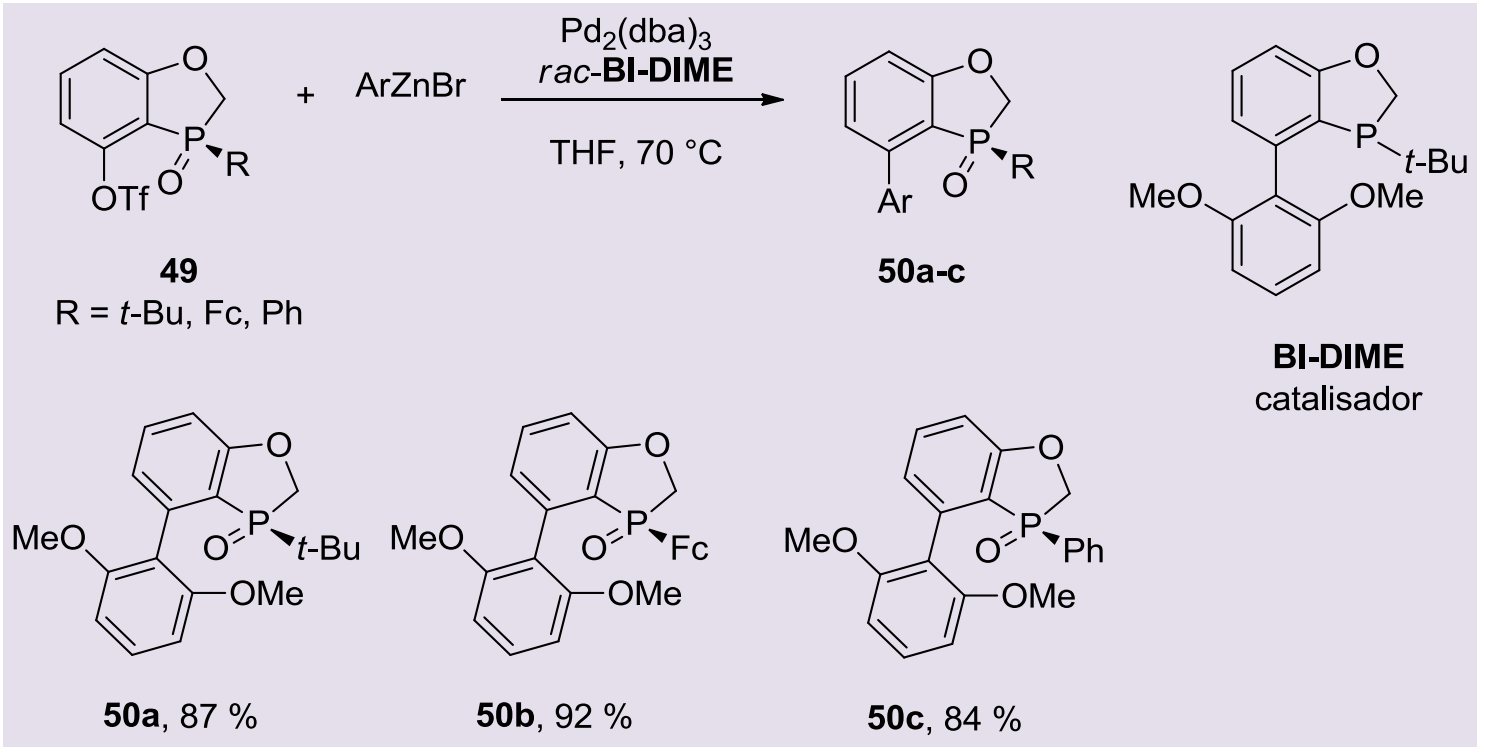

Esquema 18. Síntese de $P$-quiral di-hidrobenzo-oxafosfóis através do acoplamento cruzado de Negishi

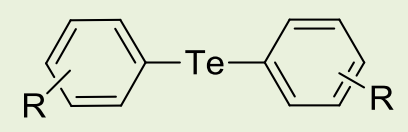

51

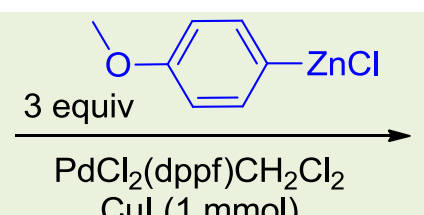

Cul $(1 \mathrm{mmol})$

THF, refluxo, $12 \mathrm{~h}$

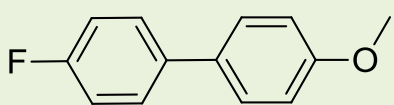

52b, $67 \%$

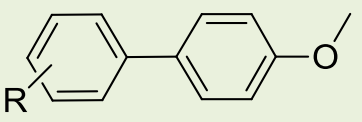

52a-c

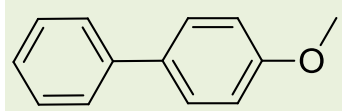

$52 a, 88 \%$

Esquema 19. Reação de acoplamento cruzado de Negishi entre diaril-teluretos e reagentes organozinco

Apesar da síntese assimétrica de biarilas utilizando o acoplamento cruzado de Negishi não ter sido explorada o suficiente, Espinet e colaboradores relataram a síntese assimétrica de binaftilas (55), reação de acoplamento cruzado de Negishi entre reagentes naftil-zinco funcionalizados (53) e bromonaftalenos (54), utilizando paládio como catalisador e um ligante quiral planar (Esquema 20). ${ }^{39}$ 


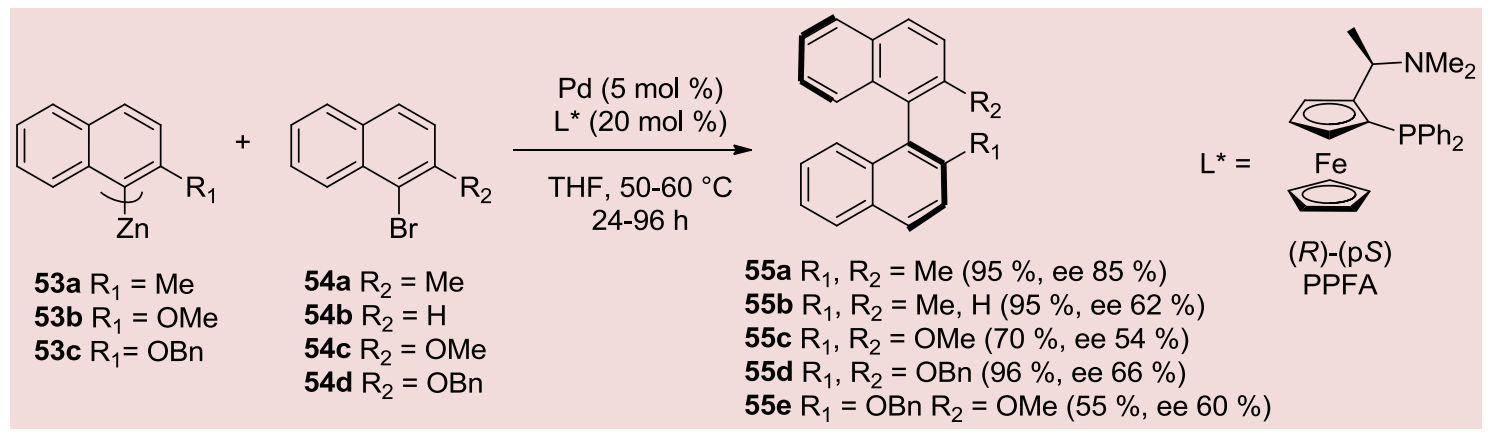

Esquema 20. Reação de acoplamento de Negishi em síntese de biarilas assimétrica

\section{Reação de Migita-Kosug-Stille}

A reação de Migita-Kosugi-Stille ${ }^{13}$ foi descoberta em 1978 e é conhecida como a reação de acoplamento cruzado catalisada com paládio entre halogenetos orgânicos e compostos organoestanados. Uma vantagem dessa reação é que os compostos de estanho não são sensíveis à umidade e ao oxigênio, no entanto, estes compostos são tóxicos. ${ }^{40}$ Além disso, essa reação tem baixa economia atômica.
Recentemente, foi descrito por Nikoorazm e colaboradores a reação de acoplamento cruzado de Migita-Kosugi-Stille de vários halogenetos de arila com $\mathrm{Ph}_{3} \mathrm{SnCl}$ (cloreto de trifenilestanho), um reagente comercialmente disponível e barato, utilizando $\mathrm{K}_{2} \mathrm{CO}_{3}$ como base, polietilenoglicol (PEG) como solvente e 0,89 mol\% de catalisador de paládio ( $\mathrm{Pd}-2 \mathrm{~A} 3 \mathrm{HP}-\mathrm{MCM}-41$ ), onde o paládio foi ancorado sobre a superfície de sílica mesoporosa (MCM-41) modificada organicamente (Esquema 21 ). ${ }^{41}$

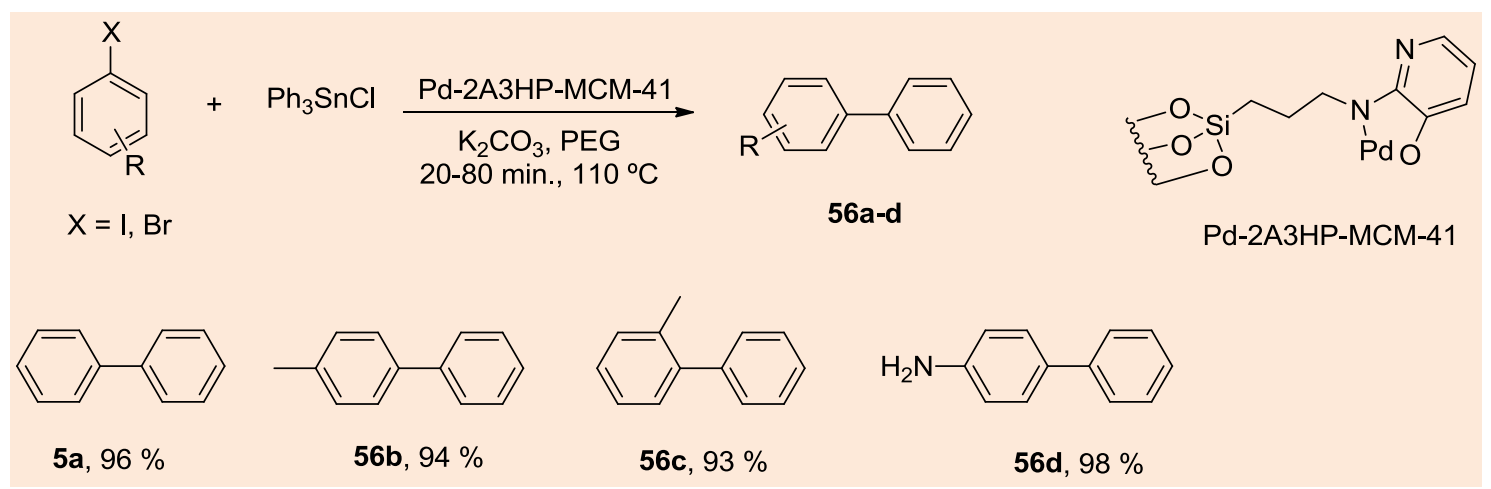

Esquema 21. Síntese de biarilas com halogenetos de arila e $\mathrm{Ph}_{3} \mathrm{SnCl}$

Em 2014, reações de acoplamento cruzado de Migita-Kosugi-Stille catalisado por $\mathrm{Pd}\left(\mathrm{PPh}_{3}\right)_{4}$ em vaso único de reação (one-pot reaction) foram descritas por Tan e colaboradores através de uma metodologia envolvendo o uso combinado de irradiação por micro-ondas e água como solvente. $\mathrm{O}$
Esquema 22 mostra a diarilação da 2,5dibromopiridina (58) com 57 e tributil(tiofen2-il)estanano e o Esquema 23 descreve a síntese da 4-clorobifenil-2-amina (61), que foi obtido a partir de dois compostos facilmente disponíveis, 1-bromo-4-clorobenzeno e 1bromo-2-nitrobenzeno. ${ }^{42}$ 


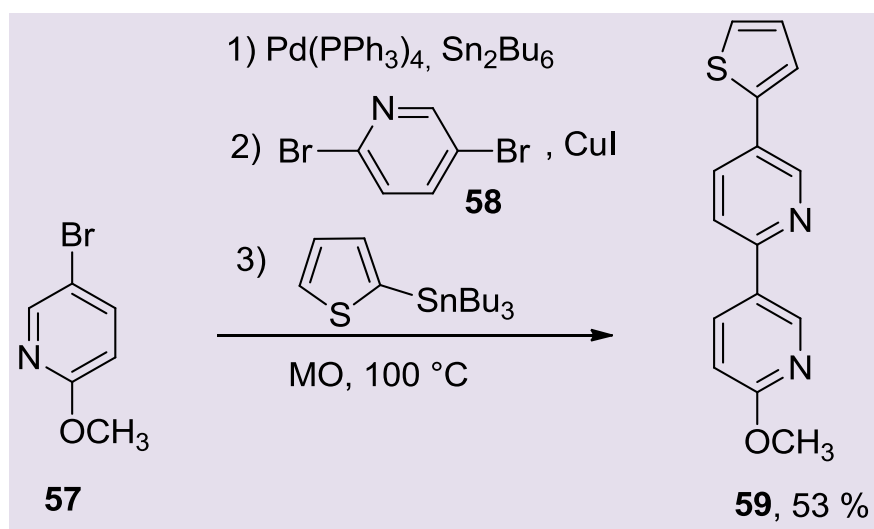

Esquema 22. Diarilação da 2,5-dibromopiridina em uma sequência one-pot

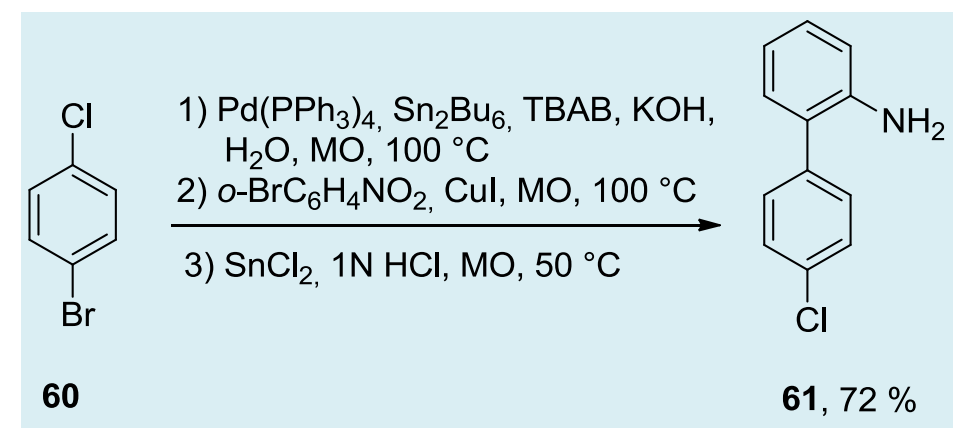

Esquema 23. Síntese da 4-clorobifenil-2-amina

Cordovilla e colaboradores descreveram a síntese de biarilas através do acoplamento cruzado de Migita-Kosugi-Stille utilizando um catalisador de paládio, um co-catalisador de ouro e trifenilfosfina como ligante em ambos os catalisadores (Esquema 24). Esta metodologia de catálise bimetálica utiliza dois metais diferentes em dois ciclos catalíticos que normalmente são interligados pela etapa de transmetalação. ${ }^{43}$

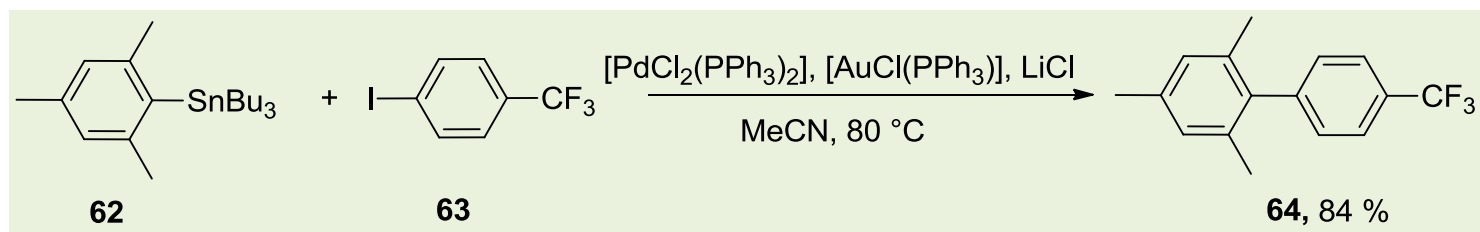

Esquema 24. Acoplamento de Migita-Kosugi-Stille em catálise bimetálica

O acoplamento cruzado de Migita-KosugiStille foi utilizado na reação de formação do intermediário macrociclo peptídeo mimético (66), intermediário-chave para a síntese total da cloropeptina I (67), com paládio como catalisador (Esquema 25). A cloropeptina é um produto natural que inibe a replicação do vírus HIV por interrupção da ligação da glicoproteína gp-120 do HIV ao receptor CD4. ${ }^{44}$ 


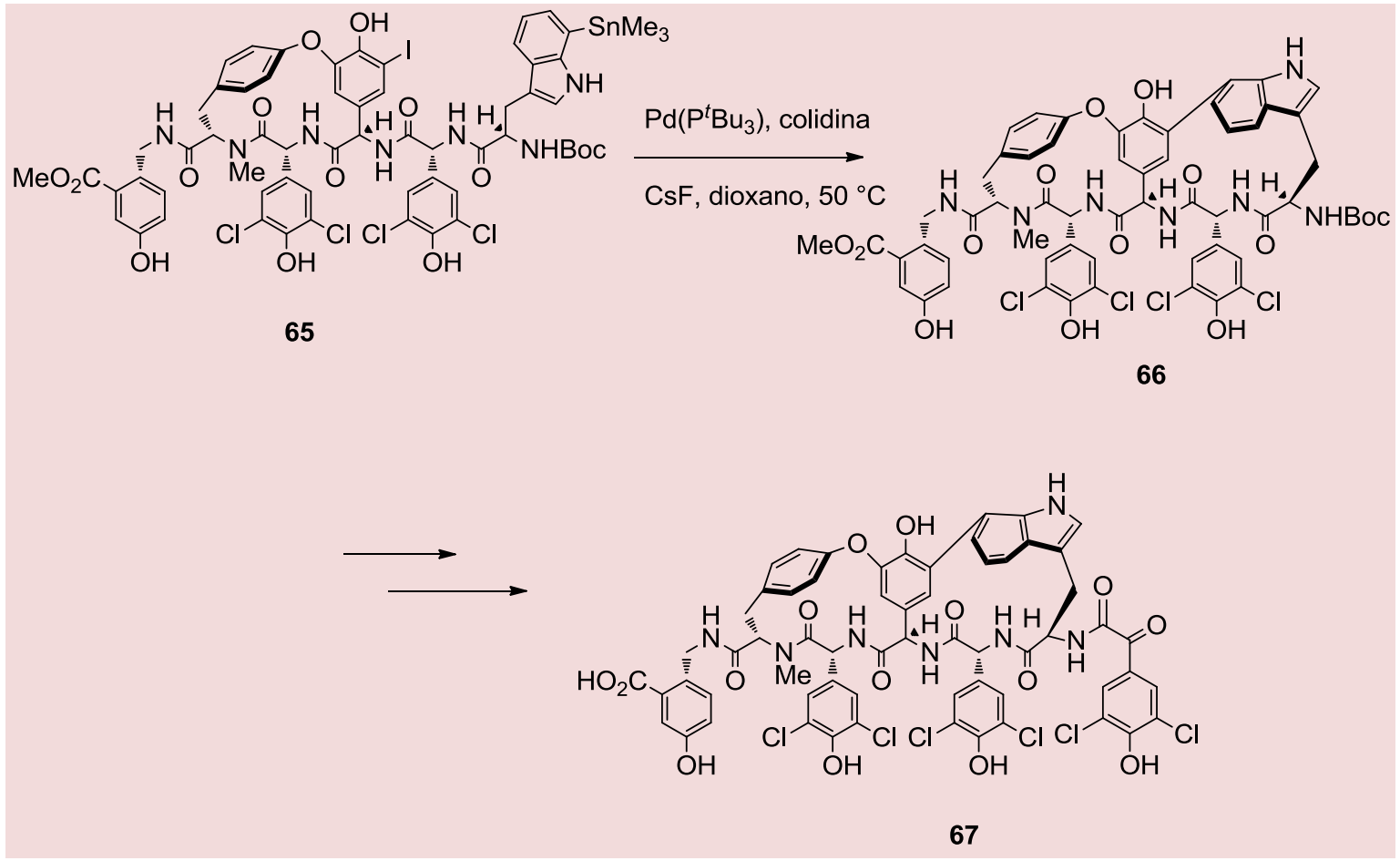

Esquema 25. Acoplamento de Migita-Kosugi-Stille empregado na síntese da cloropeptina I

Córsico e Rossi descreveram a reação de acoplamento de Migita-Kosugi-Stille entre o reagente fenil triestanado (68) e três equivalentes de iodobenzeno (69) catalisada por $\mathrm{Pd}\left(\mathrm{PPh}_{3}\right)_{2} \mathrm{Cl}_{2}$, obtendo-se o produto (70) com $89 \%$ de rendimento (Esquema 26). ${ }^{45}$<smiles>COc1cc(SC)cc(S(C)(C)c2cc([SnH3])cc(S(C)(C)C)c2)c1</smiles>

Esquema 26. Reação de acoplamento de Migita-Kosugi-Stille com o reagente triestanado

\section{Reação de Suzuki-Miayura}

A reação de Suzuki-Miyaura consiste no acoplamento entre um haleto ou um triflato com uma espécie organoborada, que geralmente são ácidos borônicos $\left(\mathrm{R}-\mathrm{B}(\mathrm{OH})_{2}\right)$. Desde a sua descoberta em $1979,{ }^{13}$ a reação de Suzuki-Miyaura é a que mais atrai atenção para os estudos nesta área. Em uma pesquisa bibliográfica no SciFinder com as palavraschave "Suzuki and biaryls", refinando para os anos de 2015 e 2016, foram encontrados 517 documentos que tratam do assunto. 0 grande impacto dessa reação pode ser explicado pela sua grande compatibilidade a uma variedade de grupos funcionais, pela sua eficiência sob diferentes condições reacionais (utilização de solventes iônicos, água, polietilenoglicóis, ou até mesmo reações sem 
solvente), além de ser adaptável a processos diferentes (micro-ondas e microtecnologias, etc.). Além disso, os derivados de boro são fáceis de serem preparados, muitos estão disponíveis comercialmente, não são tóxicos, são estáveis ao ar e à umidade, permitindo a sua manipulação sem precauções especiais. No entanto, possuem baixa economia atômica. ${ }^{20,46}$
O fungicida boscalida (74) foi produzido e introduzido no mercado pela BASF em 2002 e a segunda de suas etapas sintéticas faz uso da reação de Suzuki-Miyaura. Primeiro ocorre a formação do ácido borônico (72), o qual, em seguida, é utilizado no acoplamento de Suzuki-Miyaura catalisado por paládio (Esquema 27). ${ }^{34}$

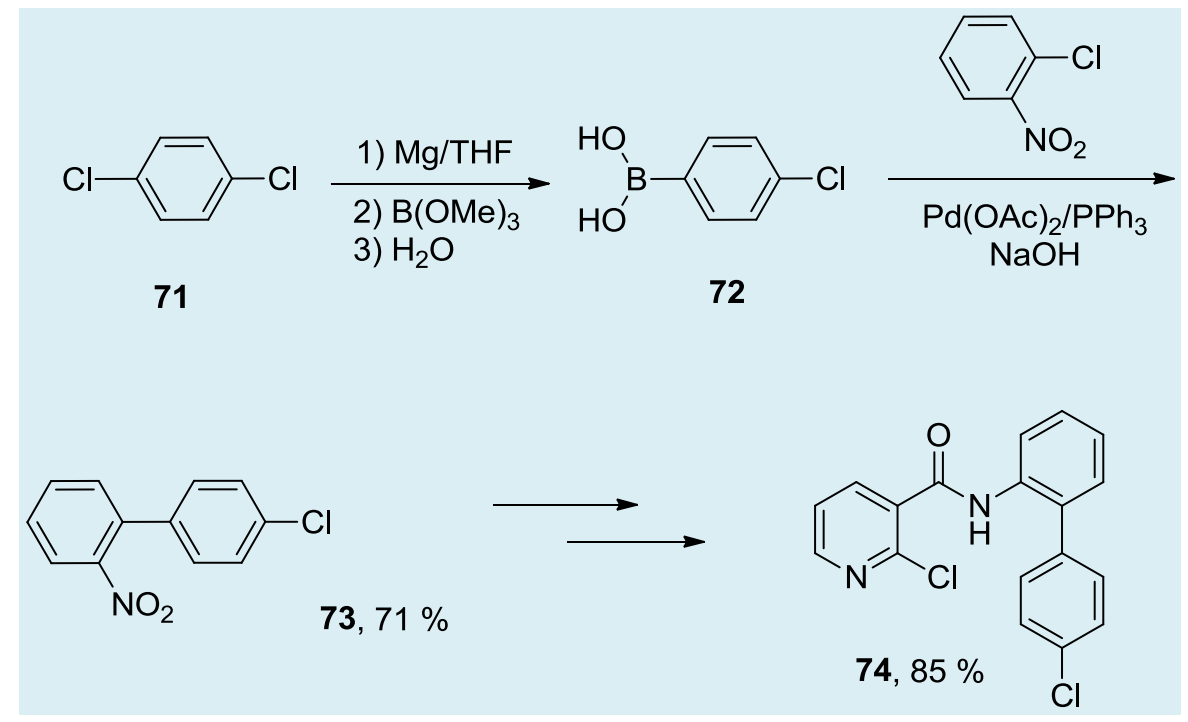

Esquema 27. Acoplamento de Suzuki-Miyaura empregado na síntese do fungicida boscalida

Recentemente, uma variedade de biarilas ou hetero-biarilas foram preparadas, com um rendimento de até $99 \%$, através do acoplamento de Suzuki-Miyaura entre arilcloretos ricos em elétrons e ácidos arilborônicos, utilizando ligante- $\left[\mathrm{Pd}_{2}(\mathrm{dba})_{3}\right]$ como catalisador (Esquema 28). ${ }^{47}$

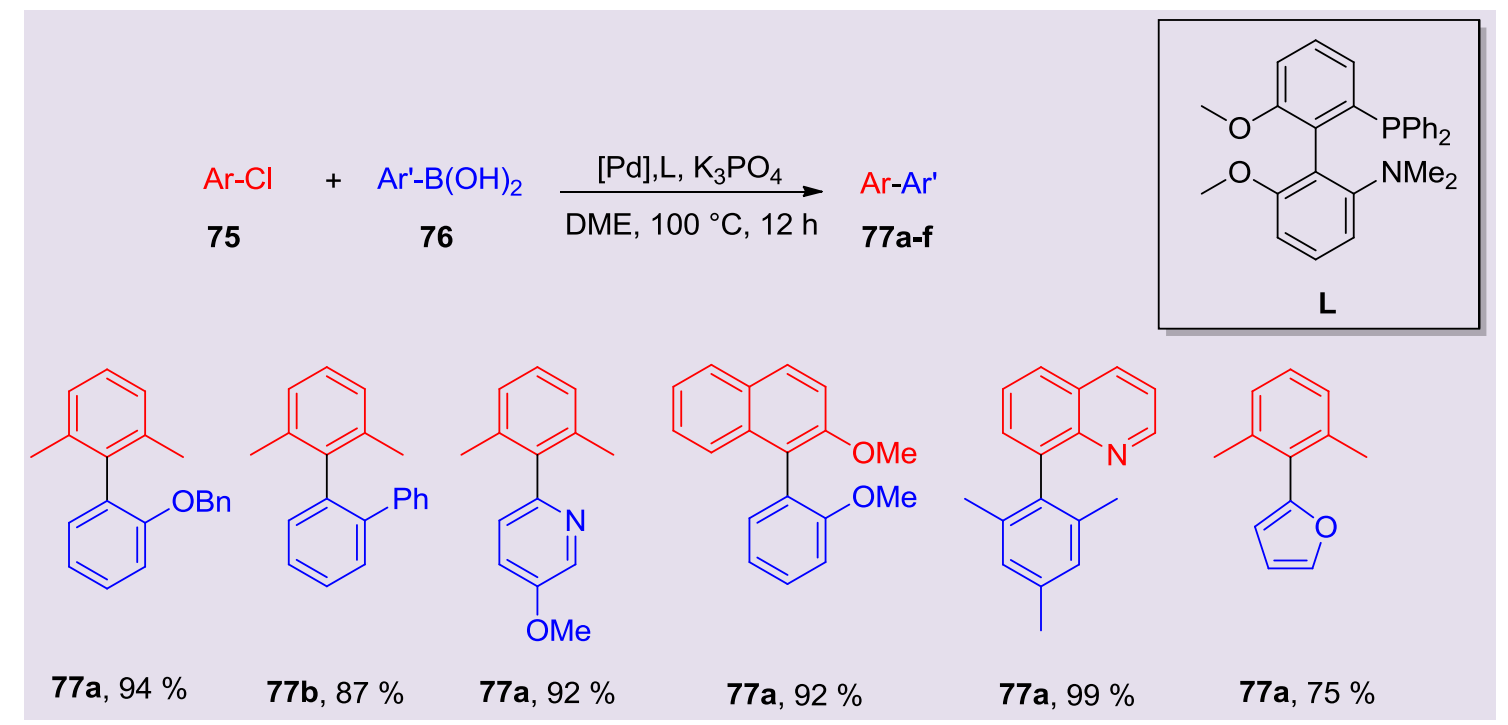

Esquema 28. Acoplamento de Suzuki-Miyaura de arilcloretos com ácidos aril borônicos 
Shi e colaboradores, recentemente desenvolveram o primeiro acoplamento de Suzuki-Miyaura de amidas por ativação da ligação N-C para a síntese de biarilas. A reação é tolerante a muitos substituintes eletronegativos e doadores de elétrons em ambos os substratos de acoplamento (derivados de amida e ácidos borônicos) e emprega-se o catalisador $\mathrm{Ni}\left(\mathrm{PCy}_{3}\right)_{2} \mathrm{Cl}_{2}$, que possui baixo custo, é estável ao ar e apresenta alta tolerância à água (Esquema 29). ${ }^{48}$

Melvin e colaboradores descreveram o acoplamento de Suzuki-Miyaura entre aril sulfamatos e ácidos borônicos, em reação catalisada por paládio e a temperatura ambiente, fornecendo biarilas com rendimentos excelentes (Esquema 30). ${ }^{49}$

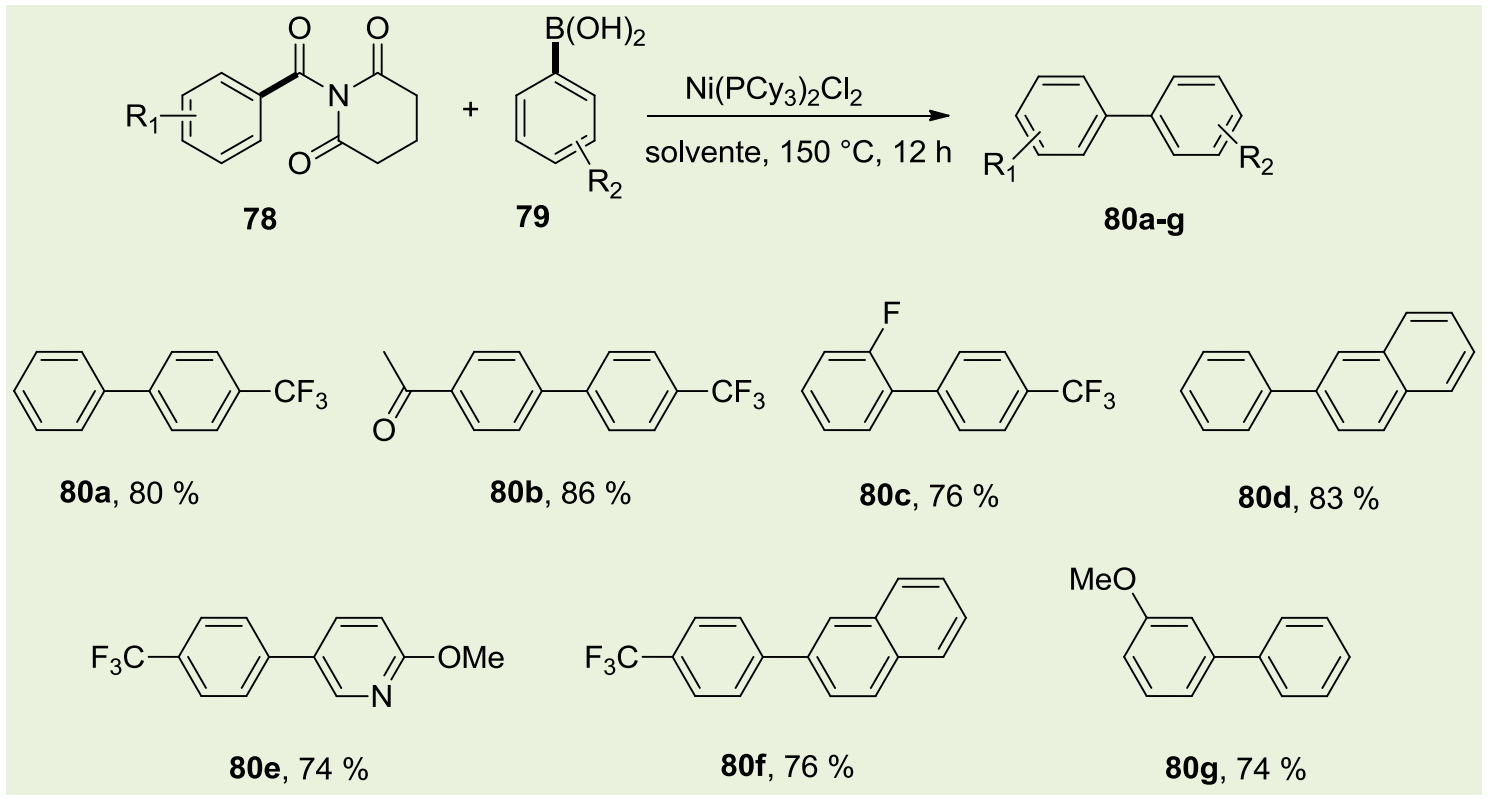

Esquema 29. Acoplamento de Suzuki-Miyaura, catalisado por níquel, entre amidas e ácidos borônicos

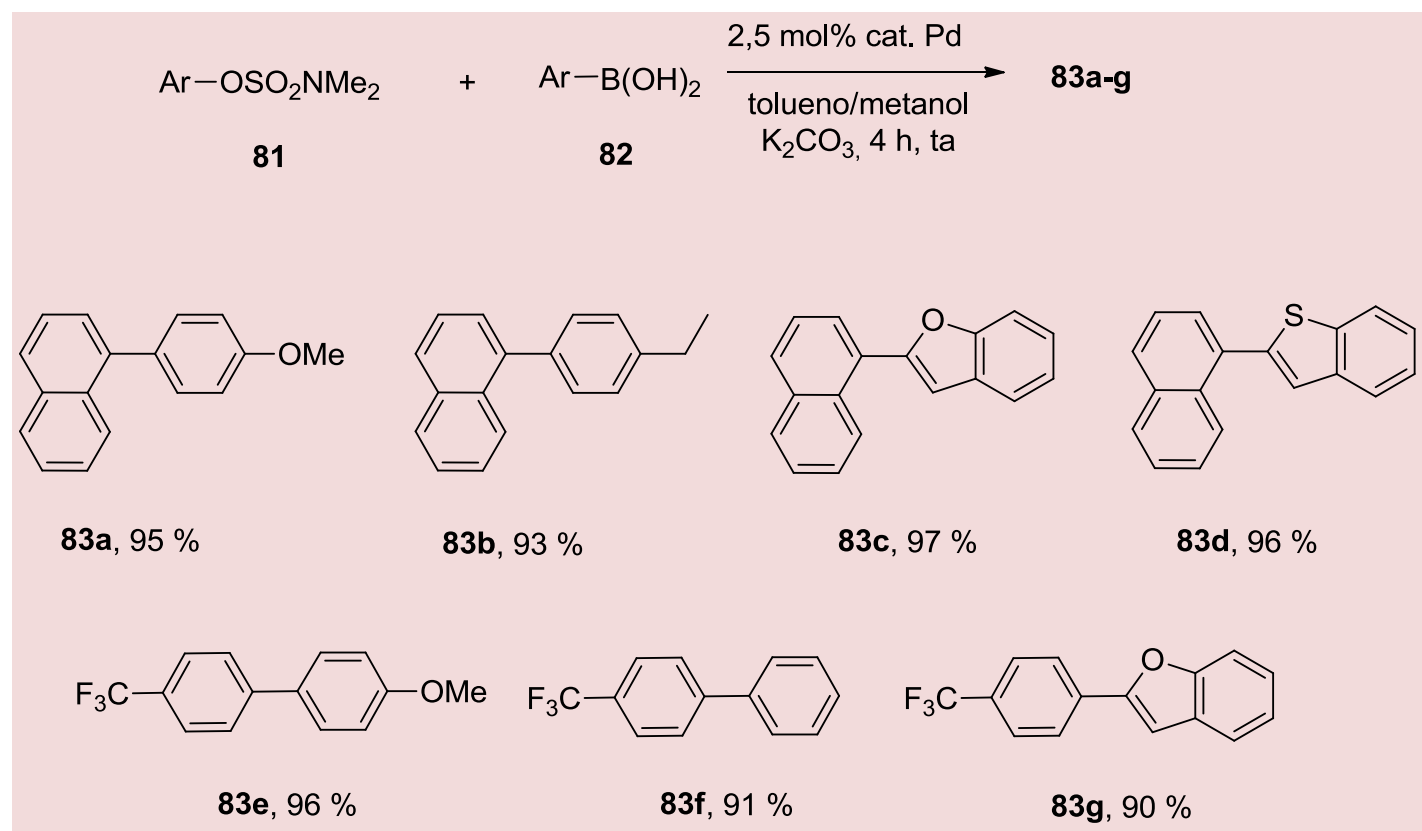

Esquema 30. Acoplamento de Suzuki-Miyaura entre aril sulfamatos e ácidos borônicos 
Handa e colaboradores descreveram o acoplamento de Suzuki-Miyaura em meio aquoso catalisado por cobre, co-catalisado por paládio, em escala ppm de quantidade
(Esquema 31). A catálise por cobre torna possível o acoplamento seletivo na presença e outros halogenetos, em particular, brometos de arila, por exemplo. ${ }^{50}$

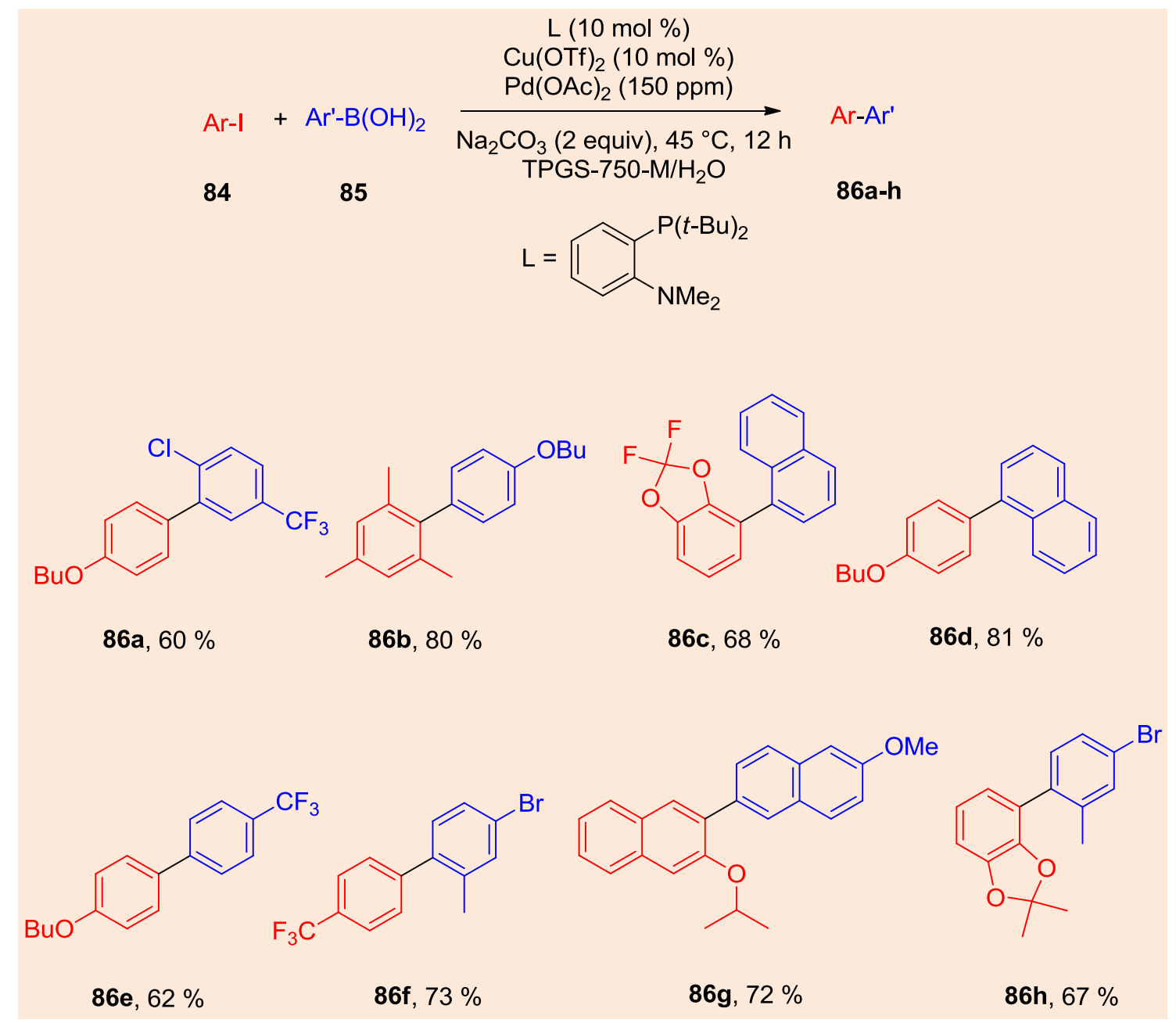

Esquema 31. Acoplamento de Suzuki-Miyaura catalisado por cobre e paládio como um cocatalisador

McNulty e colaboradores, realizaram um estudo de síntese, caracterização e avaliação de atividade biológica de dois compostos biarilas (89a-b), que apresentaram propriedades anti-proliferativas e pró- apoptóticas em várias linhagens de células cancerígenas. Os dois compostos foram facilmente obtidos através do acoplamento de Suzuki-Miyaura entre brometos de arila e ácido fenilborônico (Esquema 32). ${ }^{51}$ 


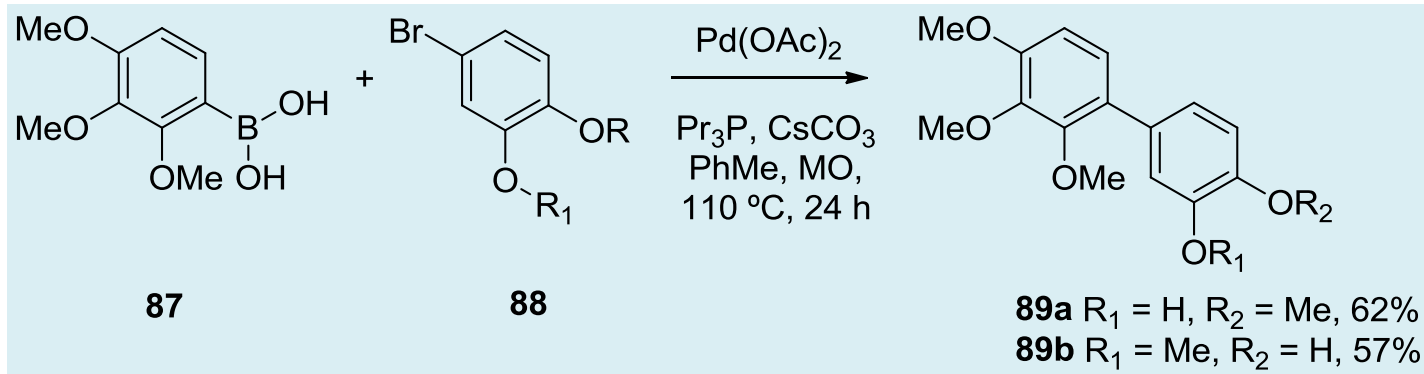

Esquema 32. Reação de acoplamento cruzado Suzuki-Miyaura de brometos de arila com ácido fenilborônico

As primeiras reações diastereoseletivas envolvendo o acoplamento de SuzukiMiyaura de halogenetos de arila com auxiliares quirais foram descritas na década de $90 .^{46}$ sintetizaram uma série de binaftilas assimétricas, através do acoplamento de Suzuki catalisado por paládio, obtendo-se produtos com boa enantiosseletividade e excelentes rendimentos (Esquema 33). ${ }^{52}$

Em 2012, Wang e colaboradores<smiles>CCOc1ccc2ccccc2c1Br</smiles>

90<smiles>COc1cccc2ccccc12</smiles>

91

$$
\begin{gathered}
\left.\underset{\mathrm{Pd}^{-}(\mathrm{dba})_{3}(2 \mathrm{~mol} \%)}{\mathrm{L}(4,8 \mathrm{~mol} \%)}\right) \\
\underset{\mathrm{K}_{3} \mathrm{PO}_{4,}(3 \text { equiv })}{\text { tolueno, } 40{ }^{\circ} \mathrm{C}, 40 \mathrm{~h}}
\end{gathered}
$$<smiles>CCOc1ccc2ccccc2c1-c1cccc2ccccc12</smiles>

92, $92-98 \%$ ee $78-97 \%$

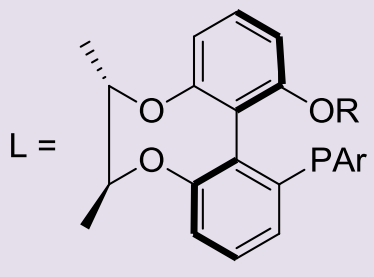

$$
\mathrm{R}=\mathrm{Me}, \mathrm{Ar}=3,5-\mathrm{tBu} \mathrm{B}_{2} \mathrm{Ph}
$$

Esquema 33. Acoplamento de Suzuki-Miyaura na síntese de binaftilas assimétricos

O acoplamento diastereosseletivo de Suzuki-Miyaura foi aplicado por Baudoin e colaboradores, na síntese do precursor (95) da esteganicina (96), uma lignana isolada da árvore Steganataenia araliacea com atividade antileucêmica (Esquema 34). ${ }^{53}$ 
<smiles>Cc1cc2c(cc1I)OCO2</smiles>

93<smiles>COCc1cc(OC)c(OC)c(Br)c1Cc1ccccc1</smiles>

94
1) $\mathrm{Pd}(\mathrm{OAc})_{2}(5 \mathrm{~mol} \%) \mathbf{L}(10 \mathrm{~mol} \%)$ $\mathrm{Ba}(\mathrm{OH})_{2} \cdot 8 \mathrm{H}_{2} \mathrm{O}$, dioxano/ $\mathrm{H}_{2} \mathrm{O}(9: 1)$

2) $\mathrm{NaH}, \mathrm{BnBr}, \mathrm{THF}, 25^{\circ} \mathrm{C}, 4 \mathrm{~h}$ $63 \%$

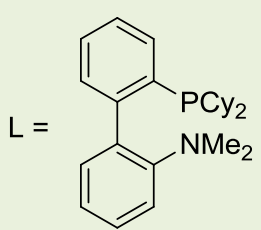

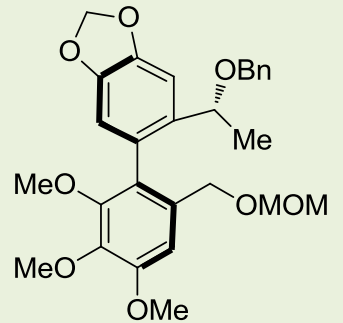

95, $53 \%$, de $68 \%$<smiles>COC(=O)C1c2cc3c(cc2-c2c(cc(OC)c(OC)c2OC)OCO3)C[C@@H]2C(=O)OCC12</smiles>

96

Esquema 34. Acoplamento de Suzuki-Miyaura empregado na síntese da esteganicina (96)

\section{Reação de Hiyama}

A reação de Hiyama trata-se do acoplamento, catalisado por sais de paládio, entre organosilanos e halogenetos orgânicos. Os organosilanos são de fácil manuseio, possuem baixa toxicidade e são bastante estáveis. ${ }^{54}$ Entretanto, esses derivados são pouco reativos, o que leva a uma necessidade de utilização de agentes de ativação ou uma fonte de íons fluoretos, para esse tipo de acoplamento. $^{20}$

Recentemente, Shi e colaboradores desenvolveram a primeira reação de acoplamento cruzado de Hiayama entre carbamatos de arila como eletrófilos e arilsilanos via catálise de níquel ou ferro (Esquema 35). Essa metodologia apresenta boa tolerância aos grupos funcionais e não é sensível ao impedimento estérico, tanto nos carbamatos de arila como nos arilsilanos. ${ }^{55}$

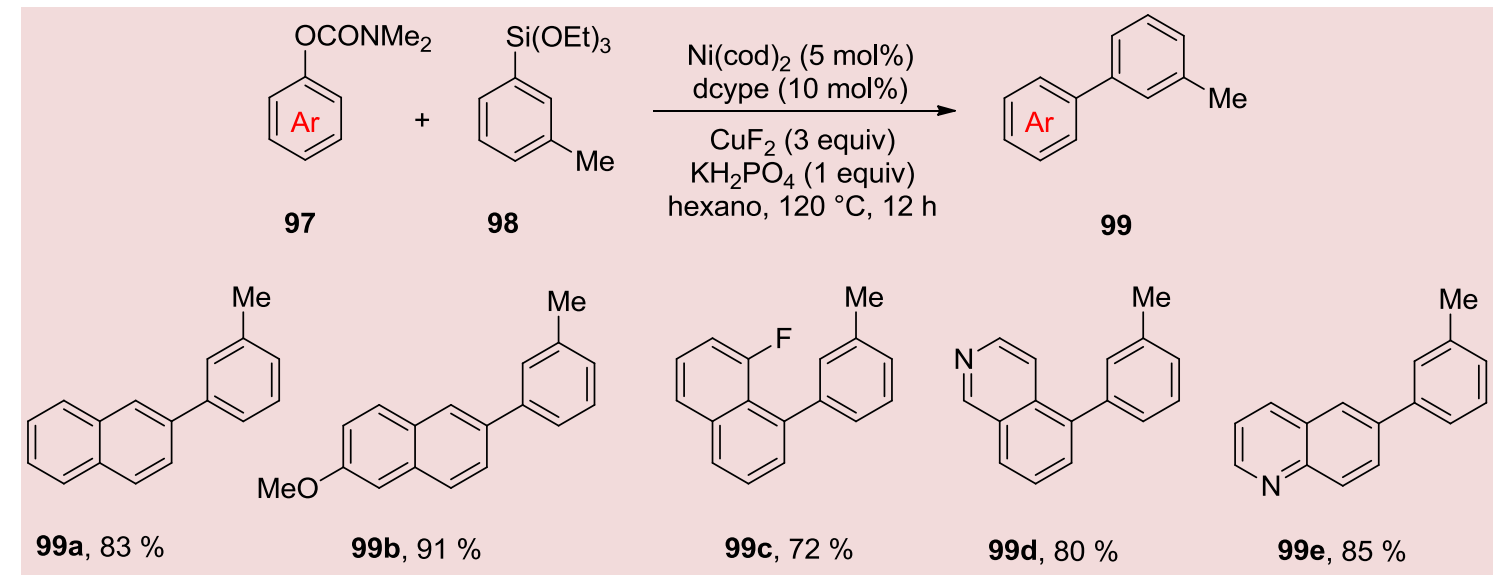

Esquema 35. Acoplamento de Hiyama entre carbamatos de arilo e arilsilanos 
Em 2016, Guo e colaboradores desenvolveram a síntese de biarilas através do acoplamento cruzado de Hiyama, catalisado por paládio, entre brometos de arila e arilsilanos (Esquema 36). Os produtos foram obtidos em rendimentos entre 83 e 92 $\%{ }^{56}$
O acoplamento cruzado de Hiyama, catalisado por paládio, entre arilhidrazinas e arilsilanos foi descrito por Zhang e colaboradores como sendo uma metodologia prática e eficaz feita em condições suaves (Esquema 37). ${ }^{57}$<smiles>[R]c1ccc(Br)cc1</smiles>

100<smiles>[R]c1ccc([SiH2]C(C)(C)C)cc1</smiles>

101

$$
\begin{gathered}
\underset{\mathrm{PEG} / \mathrm{H}_{2} \mathrm{O}(2 / 2 \mathrm{~mL})}{\mathrm{PdCl} \mathrm{L}_{2}, \mathrm{NaOH}} \\
80-100{ }^{\circ} \mathrm{C}, 2 \mathrm{~h}
\end{gathered}
$$<smiles>CC(C)N(C(C)C)P(OCc1ccccc1)OCc1ccccc1</smiles>

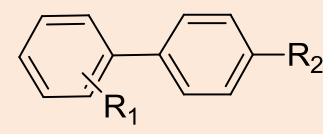

102a-d

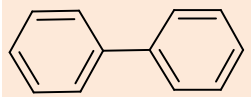

$102 a, 88 \%$

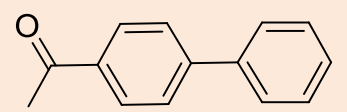

102b, $92 \%$

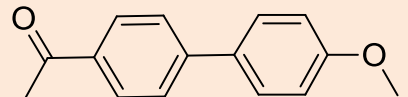

102c, $85 \%$

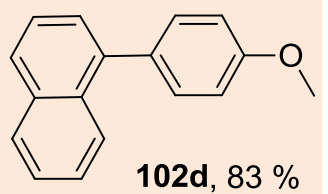

Esquema 36. Acoplamento cruzado de Hiyama, catalisado por complexo de paládio, entre brometos de arilo e arilsilanos

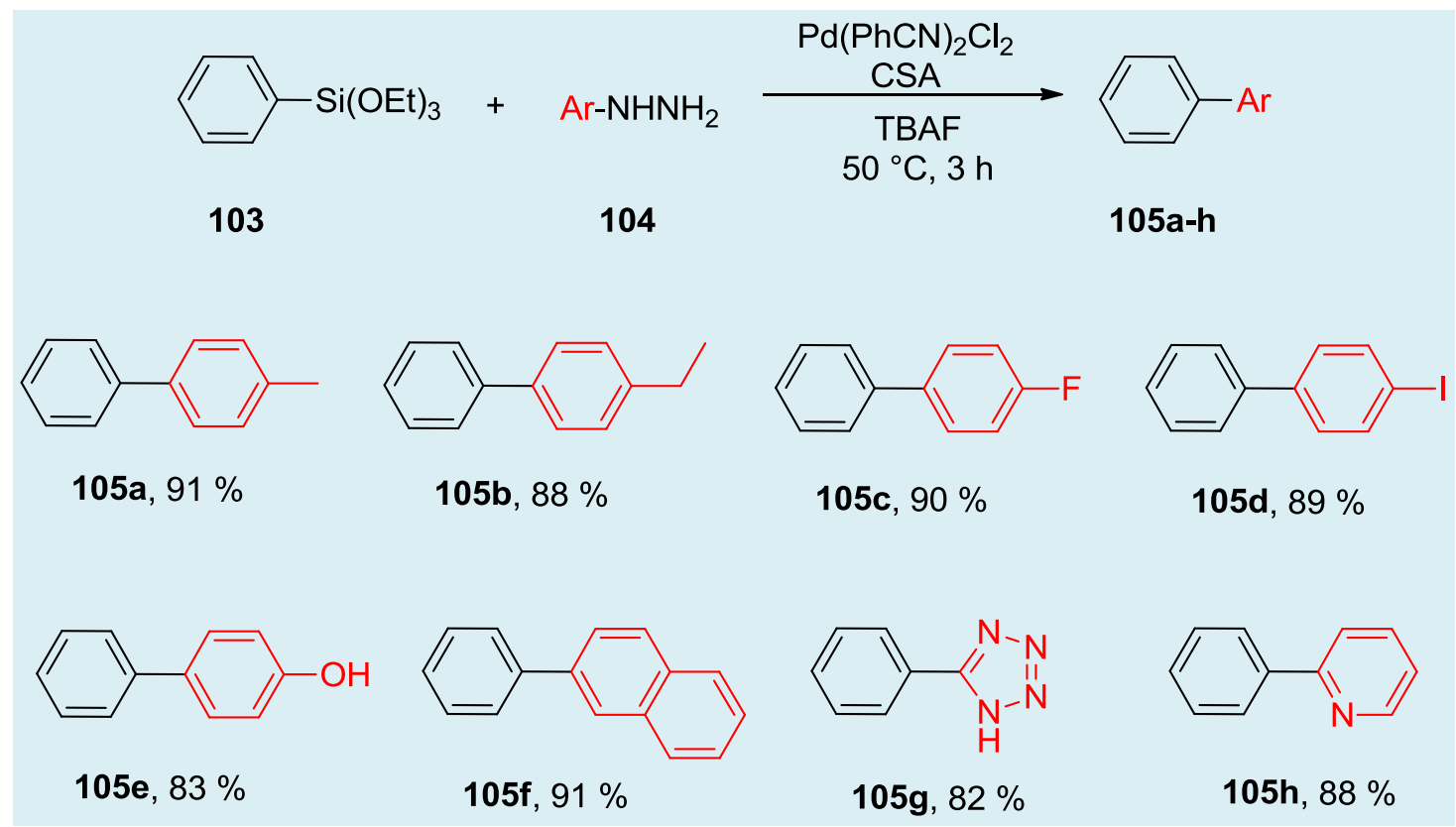

Esquema 37. $\mathrm{O}$ acoplamento cruzado de Hiyama entre arilhidrazinas e arilsilanos 
Diferentes arilsulfonil hidrazidas foram utilizadas como reagentes de acoplamento cruzado de Hiyama com trimetoxifenilsilano, obtendo-se uma série de biarilas em bons rendimentos (Esquema 38 ). ${ }^{58}$

O acoplamento de Hiyama de aril carboxilatos orto-substituídos com trialcoxiarilsilanos foi descrito por Katayev e colaboradores. A reação foi catalisada por um complexo de carbeno $\mathrm{Pd} / \mathrm{N}$-heterocíclico na presença de carbonato de prata (Esquema 39). ${ }^{59}$

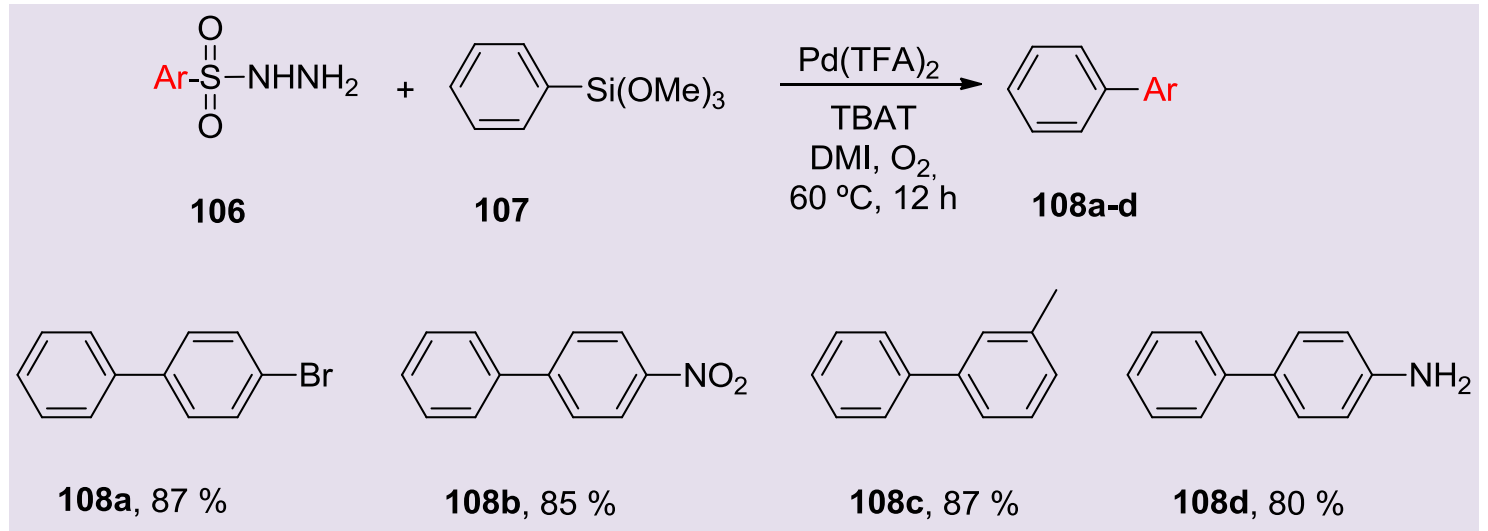

Esquema 38. Acoplamento de Hiyama entre arilsulfonil hidrazidas e trimetoxifenilsilano<smiles>[R][R]1ccc(C(=O)OC(C)C)c([N+](=O)[O-])c1</smiles><smiles>O=[N+]([O-])c1ccccc1-c1ccccc1</smiles><smiles>Cc1cccc(-c2ccccc2)c1[N+](=O)[O-]</smiles><smiles>COc1ccc([N+](=O)[O-])c(-c2ccccc2)c1</smiles><smiles>Cc1cccc([N+](=O)[O-])c1-c1ccccc1</smiles>

$111 a, 90 \%$ $111 b, 93 \%$ $111 c, 92 \%$ $111 d, 93 \%$<smiles>CN(C)c1ccc(-c2ccccc2[N+](=O)[O-])cc1</smiles>

$111 e, 97 \%$<smiles>Cc1ccc(-c2ccccc2[N+](=O)[O-])cc1</smiles>

111f, $93 \%$<smiles>O=[N+]([O-])c1ccccc1-c1ccc2c(c1)OCO2</smiles>

$111 g, 95 \%$

Esquema 39. Acoplamento de Hiyama de aril carboxilatos orto-substituídos com trialcoxiarilsilanos 


\section{Conclusão}

Neste artigo, foram mostrados alguns exemplos recentes de reações de acoplamento cruzado de Kuma-TamaoCorriu, Negishi, Migita-Kosug-Stille, SuzukiMiyaura e Hiyama como importantes ferramentas sintéticas e bastante utilizadas atualmente na química orgânica para a síntese de biarilas, os quais são de grande importância da indústria química e farmacêutica. Atualmente, muitos estudos vêm aprimorando estas reações através da introdução de diversos sistemas catalíticos, modificação de catalisadores para torná-los mais seletivos, além do uso de diferentes agentes de arilação em alternativa aos tradicionais halogenetos de arila. Para algumas dessas reações, a baixa economia atômica e a necessidade de minimizar a geração de subprodutos ainda são questões a serem superadas a fim de torná-las mais sustentáveis.

\section{Referências Bibliográficas}

${ }^{1}$ Dahlgren, M. K.; Schyman, P.; Tirado-Rives, J.; Jorgensen, W. L. Characterization of Biaryl Torsional Energetics and its Treatment in OPLS All-Atom Force Fields. Journal of Chemical Information and Modeling 2013, 53, 1191. [CrossRef]

${ }^{2}$ Hatakeyama, T.; Hashimoto, H.; Ishizuka, K.; Nakamura, M. Highly Selective Biaryl CrossCoupling Reactions between Aryl Halides and Aryl Grignard Reagents: A New catalyst Combination of $\mathrm{N}$-Heterocyclic Carbenes and Iron, Cobalt, and Nickel Fluorides. Journal of the American Chemical Society 2009, 131, 11949. [CrossRef]

${ }^{3}$ Yamaguchi, J. Em Metal-Catalyzed CrossCoupling Reactions and More; Yamaguchi, J.; Itami, K., eds.; cap 7, 2013. [CrossRef]

${ }^{4}$ Crini, D. J.; Duncia, J. V.; Aldrich, P. E.; Chiu, A. T.; Johnson, A. L.; Pierce, M. E.; Price, W. A.; Santella, J. B.; Wells, G. J.; Wexler, R. R.; Wong, P. C.; Yoo, S.-E.; Timmermans, P. B. M.
W. M. Nonpeptide Angiotensin II Receptor Antagonists: The Discovery of a Series of $\mathrm{N}$ (Biphenylylmethyl)imidazoles as Potent, Orally Active Anthypertensives. Journal of Medicinal Chemistry 1991, 34, 2525. [CrossRef] [PubMed]

${ }^{5}$ Hosie, G.; Bird, H. The topical NSAID felbinac versus oral NSAIDS: a critical review. European Journal of Rheumatology and Inflammation 1994, 14, 21. [PubMed]

${ }^{6}$ Drininger, M. W.; Druker, B. J. Specific Targeted Therapy of Chronic Myelogenous Leukemia With Imatinib. Pharmacologicals Reviews 2003, 55, 401. [CrossRef] [PubMed]

${ }^{7}$ Levine, D. P. Vancomycin: a history. Clinical infectious diseases: an official publication of the Infectious Diseases Society of America 2006, 42, 5. [CrossRef] [PubMed]

${ }^{8}$ Chen, L.; Gao, Z.; Li, Y. Immobilization of $\mathrm{Pd}$ (II) on MOFs as a highly active heterogeneous catalyst for Suzuki-Miyaura and Ullmann-type coupling reactions. Catalysis Today 2015, 245, 122. [CrossRef]

${ }^{9}$ Tamao, K., Sumitani, K., and Kumada, M. Selective carbon-carbon bond formation by cross-coupling of Grignard reagents with organic halides. Catalysis by nickel-phosphine complexes. Journal of the American Chemical Society 1972, 94, 4374. [CrossRef]

${ }^{10}$ Corriu, R. J. P. and Masse, J. P. Activation of Grignard reagents by transition-metal complexes. A new and simple synthesis of trans-stilbenes and polyphenyls. Journal of the Chemical Society, Chemical Communications 1972, 144. [CrossRef]

${ }^{11}$ (a) Baba, S.; Negishi, E. A novel stereospecific alkenyl-alkenyl cross-coupling by a palladium- or nickel-catalyzed reaction of alkenylalanes with alkenyl halides. Journal of the American Chemical Society 1976, 98, 6729; [CrossRef] (b) King, A.; Okukado, N.; Negishi, E. Highly general stereo-, region-, and chemo- selective synthesis of terminal and internal conjugated enynes by the Pdcatalyzed reaction of alkynylzinc reagents with alkenyl halides. Journal of the Chemical Society, Chemical Communications 1977, 683; [CrossRef] (c) King, A. O., Negishi, E., Villani, F. J. Jr., Silveira, A. Jr. A general synthesis of 
terminal and internal arylakynes by the palladium-catalyzed reaction of alkynylzinc reagents with aryl halides. The Journal of Organic Chemistry 1978, 43, 358. [CrossRef]

${ }^{12}$ Milstein, D.; Stille, J. K. A general, selective, and facile method for ketone synthesis from acid chlorides and organotin compounds catalyzed by palladium. Journal of the American Chemical Society 1978, 100, 3636. [CrossRef]

${ }^{13}$ (a) Miyaura, N.; Suzuki, A. Stereoselective synthesis of arylated $€$-alkenes by the reaction of alk-1-enylboranes with aryl halides in the presence of palladium catalyst. Journal of the Chemical Society, Chemical Communications 1979, 866; [CrossRef] (b) Miyaura, N.; Yanagi, T.; Suzuki, A. The Palladium-Catalyzed Cross-Coupling Reaction of Phenylboronic Acid with Haloarenes in the Presence of Bases. Synthetic Communications 1981, 11, 513. [CrossRef]

${ }^{14}$ Hatanaka, Y.; Hiyama, T. Highly Selective Cross-Coupling Reactions of Organosilicon Compounds Mediated by Fluoride Ion and a Palladium Catalyst. Synlett 1991, 845. [CrossRef]

${ }^{15}$ Li, C.; Chen, D.; Tang, W. Addressing the Challenges in Suzuki-Miyaura Cross-Couplings by Ligand Design. Synlett 2016, 2183. [CrossRef]

${ }^{16}$ Yamaguchi, J.; Yamaguchi, A. D.; Itami, K. C$\mathrm{H}$. Bond Functionalization: Emerging Synthetic Tools for Natural Products and Pharmaceuticals. Angewandte Chemie International Edition 2012, 51, 2. [CrossRef] [PubMed]

${ }^{17}$ Gao, H.; Ess, D. H.; Yousufuddin, M.; Kurti, L. Transition-Metal-Free Direct Arylation: Synthesis of Halogenated 2-Amino-2hydroxy-1,1-biaryls and Mechanism by DFT Calculations. Journal of the American Chemical Society 2013, 135, 7086. [CrossRef] [PubMed]

${ }^{18}$ Martins, D. L. Acoplamentos de KumadaCorriu-Tamao Catalisados por $\mathrm{Ni}$ e $\mathrm{Pd}$ : Uma Ferramenta Importante para a Síntese de Biarilas. Revista Virtual de Química 2010, 2 (4), 231. [CrossRef]
${ }^{19}$ Ren, G.; Cui, X.; Wu, Y. Efficient Synthesis of Biaryls through the Kumada Reaction Catalyzed by Carbene Adducts of Cyclopalladated Ferrocenylimine. European Journal of Organic Chemistry 2010, 2372. [CrossRef]

${ }^{20}$ Gurbuz, N.; Karaca, E. O.; Ozdemir, I.; Çetinkaya, B. Cross coupling reactions catalyzed by (NHC)Pd(II) complexes. Turkish Journal of Chemistry 2015, 39, 1115. [CrossRef]

${ }^{21}$ (a) Kochi, J. K.; Tamura, M. Mechanism of the silver-catalyzed reaction of grignar reagents with alkyl halides. Journal of the American Chemical Society 1971, 93, 1483; [CrossRef] (b) Kochi, J. K.; Tamura, M. Alkylcopper(I) in the coupling of Grignar reagents with alkyl halides. Journal of the American Chemical Society 1971, 93, 1485; [CrossRef] (c) Tamura, M.; Kochi, J. K. Vinylation of Grignar reagents. Catalysis by iron. Journal of the American Chemical Society 1971, 93, 1487. [CrossRef]

${ }^{22}$ Minato, A.; Suzuki, K.; Tamao, K.; Kumada, M. Mixed heteroarene oligomers. Journal of the Chemical Society, Chemical Communications 1984, 511. [CrossRef]

${ }^{23}$ Noyori, S.; Nishihara, Y. Recent Advances in Cross-Coupling Reactions with Aryl Chlorides, Tosylates and Mesylates. Applied CrossCoupling Reactions 2012, 80, 177. [CrossRef]

${ }^{24}$ Zhang, S.; Li, X.; Sun, H.; Fuhr, O.; Fenske, D. Nickel(II) complexes of amine functionalized $\mathrm{N}$-heterocyclic carbenes (NHCs), synthesis and catalysis in Kumada coupling of aryl chlorides. Journal of Organometallic Chemistry 2016, 820, 41. [CrossRef]

${ }^{25}$ Kuchtanin, V.; Klesciková, L.; Soral, M.; Fischer, R.; Ruzicková, Z.; Rakovsky, E.; Moncol, J.; Segl'a, P. Nickel (II)Schiff base complexes: Synthesis, characterization and catalytic activity in Kumada-Corriu crosscoupling reactions. Polyhedron 2016, 117, 90. [CrossRef]

${ }^{26}$ Fan, X.; Song, Y-L.; Long, Y-Q. An Efficient and Pratical Synthesis of the HIV Protease 
Inhibitor Atazanavir via a Highly Diastereoselective Reduction Approach. Organic Process Research \& Development 2008, 12, 69. [CrossRef]

${ }^{27}$ Stein, A. L.; Bilheri, F. N.; Zeni, G. Application of organoselenides in the Suzuki, Negishi, Sonogashira and Kumada crosscoupling reactions. Chemical Communication 2015, 51, 15522. [CrossRef] [PubMed]

${ }^{28}$ Srinivas, J.; Singh, P. P.; Varma, Y. K.; Hyder, I.; Kumar, H. M. S. Concise total synthesis of honokiol via Kumada cross coupling. Tetrahedron Letters 2014, 55, 4295. [CrossRef]

${ }^{29}$ Ackermann, L.; Kapdi, A. R.; Fenner, S.; Kornhaab, C.; Schulzke, C. Well-Defined AirStable Palladium HASPO Complexes for Efficient Kumada-Corriu Cross-Coupling of (Hetero)Aryl or Alkenyl Tosylates. Chemistry a European Journal 2011, 17, 2965. [CrossRef]

30 Tamao, K; Minato, A.; Miyake, N.; Matsuda, T.; Kiso, Y.; Kumada, M. Nickel-Phosphine Complex Catalyzed Grignard Synthesis of Sterically Hindered, Unsymmetrical Biaryls: An Approach to the Asymmetric Synthesis of Biaryl Atropisomers. Chemistry Letters 1975, 4, 133. [CrossRef]

${ }^{31}$ Wu, L.; Salvador, A.; Ou, A.; Shi, M. W.; Skelton, B. W.; Dorta, R. Monodentate Chiral $\mathrm{N}$-Heterocyclic Carbene-Palladium-Catalyzed Asymmetric Suzuki-Miyaura and Kumada Coupling. Synlett 2013, 1215. [CrossRef]

${ }^{32}$ Wang, M.; Liu, F.; Zeng, M-T.; Xu, W.; Liu, M.; Dong, Z-B. A cross-coupling synthesis of functionalised biaryls using Knochel-type organizing reagents and a pyridine enhanced palladium catalyst. Journal of Chemical Research 2016, 40, 382. [CrossRef]

${ }^{33}$ Roster, S.; Buchwald, S. L. Continuous-Flow Synthesis of Biaryls by Negishi Cross-Coupling of Fluoro- and Trifluoromethyl-Substituted (Hetero)arenes. Angewandte Chemie International Edition 2016, 55, 1. [CrossRef] [PubMed]

${ }^{34}$ Vries, J. G. Palladium-Catalysed Coupling Reactions. Topics in Organometallic Chemistry 2012, 42, 1. [CrossRef]
${ }^{35}$ Wu, W-Y.; Lin, T-C.; Takahashi, T.; Tsai, F-Y.; Mou, C-Y. A palladium Bipyridyl Complex Grafted onto nanosized MCM-41 as a Heterogeneous Catalyst for Negishi Coupling. ChemCatChem 2013, 5, 1011. [CrossRef]

${ }^{36}$ Thapa, S.; Vangala, A. S.; Giri, R. CopperCatalyzed Negishi Coupling of Diarylzinc Reagents with Aryl lodides. Synthesis 2016, 504. [CrossRef]

${ }^{37}$ Sieber, J. D.; Qu, B.; Rodríguez, S.; Haddad, N.; Grinberg, N.; Lee, H.; Song, J. J.; Yee, N. K.; Senanayake, C. H. Synthesis of P-Chiral Dihydrobenzooxaphospholes Through Negishi Cross-Coupling. The Journal of Organic Chemistry 2015, 81, 729. [CrossRef] [PubMed]

${ }^{38}$ Stefani, H. A.; Pena, J. M.; Manarin, F.; Ando, R. A.; Leal, D. M.; Petragnani, N. Negishi cross-coupling of organotellurium compounds: synthesis of biaryls, aryl-, and diaryl acetylenes. Tetrahedron Letters 2011, 52, 4398. [CrossRef]

39 (a) Genov, M.; Fuents, B.; Espinet, P.; Pelaz, B. Asymmetric Negishi reaction for sterically hindered couplings: synthesis of chiral binaphthalenes. Tetrahedron: Asymmetry 2006, 17, 2593; [CrossRef] (b) Genov, M.; Almorín, A.; Espinet, P. Microwave assisted asymmetric SuzukiMiyaura and Negishi cross-coupling reactions: synthesis of chiral binaphthalenes. Tetrahedron: Asymmetry 2007, 18, 625. [CrossRef]

${ }^{40}$ Cordovilla, C.; Bartolomé, C.; MartínezIlarduya, J. M.; espinet, P. The Stille Reaction, 38 Years Later. ACS Catalysis 2015, 5, 3040. [CrossRef]

${ }^{41}$ Nikoorazm, M.; Ghorbani-Choghamarani, A.; Khanmoradi, M. Application of Pd-2A3HPMCM-41 to the Suzuki, Heck and Stille coupling reactions and synthesis of 5substituted $1 \mathrm{H}$-tetrazoles. Applied Organometallic Chemistry 2016, 30, 705. [CrossRef]

${ }^{42}$ Tan, X.; Zhou, Z. J.; Zhang, J. X.; Duan, X. H. Efficient One-Pot Cross-Coupling of Two Aryl Halides by Stannylation/Stille Reaction in Water under Microwave Irradiation. 
European Journal of Organic Chemistry 2014, 5153. [CrossRef]

${ }^{43}$ Cordovilla, C.; Bartolomé, C.; MartínezIlarduya, J. M.; espinet, P. The Stille Reaction, 38 Years Later. ACS Catalysis 2015, 5, 3040. [CrossRef]

${ }^{44}$ Deng, H.; Jung, J-K.; Liu, T.; Kuntz, K. W.; Snapper, M. L.; Hoveyda, A. H. Total synthesis of anti-HIV agent chloropeptin I. Journal of the American Chemical Society 2003, 125, 9032. [CrossRef] [PubMed]

${ }^{45}$ Córsio, E. F.; Rossi, R. A. Synthesis of Mono, Di-, and Tri-Phenyl Arenes by Sequential Photostimulated $\mathrm{S}_{\mathrm{RN}} 1$ and $\mathrm{Pd}(0)$-Catalyzed Cross Coupling. Synlett 2000, 230. [CrossRef]

${ }^{46}$ Loxq, P.; Manoury, E.; Poli, R.; Deydier, E.; Labande, A. Synthesis of axially chiral biaryl compounds by asymmetric catalytic reactions with transition metals. Coordination Chemistry Reviews 2016, 308, 131. [CrossRef]

${ }^{47}$ Zhou, Z.; Zhang, Y.; Xia, W.; Chen, H.; Liang, H.; He, X.; Yu, S.; Cao, R.; Qiu, L. PalladiumCatalyzed Suzuki-Miyaura Coupling Reactions of Boronic Acid Derivatives with Ary Chlorides. Asian Journal of Organic Chemistry 2016, 5, 1260. [CrossRef]

${ }^{48}$ Shi, S.; Meng, G.; Szostak, M. Synthesis of Biaryls through Nickel-Catalyzed SuzukiMiyaura Coupling of Amides by CarbonNitrogen Bond Cleavage. Angewandte Chemie International Edition 2016, 55, 6959. [CrossRef]

49 Melvin, P. R.; Hazari, N.; Beromi, M. M.; Shah, H. P.; Williams, M. J. Pd-Catalyzed Suzuki-Miyaura and Hiyama-Denmark Couplings of Aryl Sulfamates. Organic Letters 2016, 18, 5784. [CrossRef] [PubMed]

${ }^{50}$ Handa, S.; Smith, J. D.; Hageman, M. S.; Gonzalez, M.; Lipshutz, B. H. Synergistic and Selective Copper/ppm Pd-catalyzed SuzukiMiyaura Couplings: In Water, Mild Conditions, with Recycling. ACS Catalysis 2016, 6, 8179. [CrossRef]

51 (a) Ullah, E.; McNulty, J.; Kennedy, C.; Robertson, $\mathrm{A}$. One step to $\mathrm{P}, \mathrm{O}$-and $\mathrm{P}, \mathrm{N}$-type heterocyclic tertiary phosphine ligands and application in Suzuki-Miyaura cross-coupling reactions. Organic and Biomolecular Chemistry 2011, 9, 4421; [CrossRef] [PubMed] (b) McNulty, J.; Berg, S. V. D.; Ma, D.; Tarade, D.; Joshi, S.; Church, J.; Pandey, S. Antimitotic activity of structurally simplified biaryl analogs of the anticancer agents colchicine and combretastatin A4. Bioorganic and Medicinal Chemistry Letters 2015, 25, 117. [CrossRef] [PubMed] (c) Tarade, D.; Ma, D.; Pignanelli, C.; Mansour, F.; Simard, D.; Berg, S. V. D.; Gauld, J.; McNulty, J.; Pandey, S. Structurally simplified biphenyl combretastatin A4 derivatives retain in vitro anti-cancer activity dependent on mitotic arrest. Plos One 2017, 12, e0171806. [CrossRef] [PubMed]

${ }^{52}$ Wang, S.; Li, J.; Miao, T.; Wu, W.; Li, Q.; Zhuang, Y.; Zhou, Z.; Qiu, L. Highly Efficient Synthesis of a Class of Novel Chiral-Bridged Atropisomeric Monophosphine Ligands via Simple Desymmetrization and Their Applications in Asymmetric Suzuki-Miyaura Coupling Reaction. Organic Letters 2012, 14, 1966. [CrossRef] [PubMed]

${ }^{53}$ Baudoin, O.; Décor, A.; Cesario, M.; Guéritte, F. A Novel 1,3-Central-to-Axial Chirality Induction Approach to Cyclooctadiene Lignans. Synlett 2003, 13, 2009. [CrossRef]

${ }^{54}$ Rostamnia, S.; Hossieni, H. G.; Doustkhah, E. Homoleptic chelating N-heterocyclic carbene complexes of palladium immobilized within the pores of SBA-15/IL (NHCePd@SBA15/IL) as heterogeneous catalyst for Hiyama reaction. Journal Organometalllic Chemistry 2015, 791, 18. [CrossRef]

${ }^{55}$ Shi, W.-J.; Zhao, H.-W.; Wang, Y.; Cao, Z.-C.; Zhang, L-S.; Yu, D-G.; Shi, Z-J. Nickel- or IronCatalyzed Cross-Coupling of Aryl Carbamates with Arylsilanes. Advanced Synthesis \& Catalysis 2016, 358, 2410. [CrossRef]

${ }^{56}$ Guo, M.; Fu, L.; Li, J.; Zhou, L.; Kang, Y. A well-defined

$\{[(\mathrm{PhCH} 2 \mathrm{O}) 2 \mathrm{P}(\mathrm{CH} 3) 2 \mathrm{CHNCH}(\mathrm{CH} 3) 2] 2 \mathrm{PdCl} 2\}$

Complex Catalyzed Hiyama Coupling of Aryl Bromides with Arylsilanes. Molecules 2016, 21, 987. [CrossRef] [PubMed] 
Silva, T. B. et al.

${ }^{57}$ Zhang, H.; Wang, C.; Li, Z.; Wang, Z. reaction of arylsufonyl hydrazides under Palladium-catalyzed denitrogenative Hiyama oxygen. Organic and Biomolecular Chemistry cross-coupling with arylhidrazines under air. 2015, 13, 4547. [CrossRef]

Tetrahedron Letters 2015, 56, 5371. ${ }^{59}$ Katayev, D.; Exner, B.; Gooben, L. J. [CrossRef] Synthesis of Biaryls by Decarboxylative

${ }^{58}$ Miao, H.; Wang, F.; Zhou, S.; Zhang, G.; Li, Hiyama Coupling. ChemCatChem 2015, 7, Y. Palladium-catalyzed Hiyama coupling 2028. [CrossRef] 